\title{
Nonlocal Cahn-Hilliard-Navier-Stokes systems with singular potentials
}

\author{
Sergio Frigeri and Maurizio Grasselli
}

Communicated by James Robinson, received February 3, 2012.

\begin{abstract}
Here we consider a Cahn-Hilliard-Navier-Stokes system characterized by a nonlocal Cahn-Hilliard equation with a singular (e.g., logarithmic) potential. This system originates from a diffuse interface model for incompressible isothermal mixtures of two immiscible fluids. We have already analyzed the case of smooth potentials with arbitrary polynomial growth. Here, taking advantage of the previous results, we study this more challenging (and physically relevant) case. We first establish the existence of a global weak solution with no-slip and no-flux boundary conditions. Then we prove the existence of the global attractor for the 2D generalized semiflow (in the sense of J.M. Ball). We recall that uniqueness is still an open issue even in $2 \mathrm{D}$. We also obtain, as byproduct, the existence of a connected global attractor for the (convective) nonlocal Cahn-Hilliard equation. Finally, in the 3D case, we establish the existence of a trajectory attractor (in the sense of V.V. Chepyzhov and M.I. Vishik).
\end{abstract}

\section{Contents}

1. Introduction 274

2. Weak solutions and existence theorem 275

3. Proof of Theorem $1 \quad 279$

4. Global attractor in 2D 288

5. Existence of a trajectory attractor 292

6. Further properties of the trajectory attractor 300

References 303

1991 Mathematics Subject Classification. 35Q30, 37L30, 45K05, 76T99.

Key words and phrases. Navier-Stokes equations, nonlocal Cahn-Hilliard equations, singular potentials, incompressible binary fluids, global attractors, trajectory attractors. 


\section{Introduction}

In [14] we have introduced and analyzed an evolution system which consists of the Navier-Stokes equations for the fluid velocity $u$ suitably coupled with a non-local convective Cahn-Hilliard equation for the order parameter $\varphi$ on a given (smooth) bounded domain $\Omega \subset \mathbb{R}^{d}, d=2,3$. This system derives from a diffuse interface model which describes the evolution of an incompressible mixture of two immiscible fluids (see, e.g., $[\mathbf{2 6}, \mathbf{2 8}, \mathbf{2 9}, \mathbf{3 0}, \mathbf{3 2}]$ and references therein). We suppose that the temperature variations are negligible and the density is constant and equal to one. Thus $u$ represents an average velocity and $\varphi$ the relative concentration of one fluid (or the difference of the two concentrations). Then the nonlocal Cahn-HilliardNavier-Stokes system reads as follows

$$
\begin{aligned}
& \varphi_{t}+u \cdot \nabla \varphi=\Delta \mu, \\
& u_{t}-\operatorname{div}(2 \nu(\varphi) D u)+(u \cdot \nabla) u+\nabla \pi=\mu \nabla \varphi+h, \\
& \mu=a \varphi-J * \varphi+F^{\prime}(\varphi), \\
& \operatorname{div}(u)=0
\end{aligned}
$$

in $\Omega \times(0,+\infty)$. We endow the system with the boundary and initial conditions

$$
\begin{aligned}
& \frac{\partial \mu}{\partial n}=0, \quad u=0, \quad \text { on } \partial \Omega, \\
& u(0)=u_{0}, \quad \varphi(0)=\varphi_{0}, \quad \text { in } \Omega,
\end{aligned}
$$

where $n$ is the unit outward normal to $\partial \Omega$. Here $\nu$ is the viscosity, $\pi$ the pressure, $h$ denotes an external force acting on the fluid mixture, $J: \mathbb{R}^{d} \rightarrow \mathbb{R}$ is a suitable interaction kernel, $a$ is a coefficient depending on $J$ (see section below for the related assumptions), $F$ is the configuration potential which accounts for the presence of two phases.

Here we prove the existence of a global weak solution when the double-well potential $F$ can be singular in $(-1,1)$, that is, its derivative is unbounded at the endpoints. A typical situation of physical interest is the following (see [10])

$$
F(s)=\frac{\theta}{2}((1+s) \log (1+s)+(1-s) \log (1-s))-\frac{\theta_{c}}{2} s^{2},
$$

where $\theta, \theta_{c}$ are the (absolute) temperature and the critical temperature, respectively. If $0<\theta<\theta_{c}$ then phase separation occurs, otherwise the mixed phase is stable. We recall that the logarithmic terms are related to the entropy of the system.

For the existence of a weak solution, we take advantage of our previous analysis for regular potentials (i.e., defined on the whole $\mathbb{R}$ ) with polynomially controlled growth of arbitrary order (see [14]) and we use a suitable approximation procedure inspired by [18]. Then, we extend to potentials like (1.7) the results obtained in [21] for regular potentials. Such results are concerned with the global longtime 
behavior of (weak) solutions. More precisely, in the spirit of [5], we can define a generalized semiflow in 2D and prove that it possesses a global (strong) attractor by using the energy identity. Then we analyze the $3 \mathrm{D}$ case by means of the trajectory approach introduced in [20] (see also [34]) and generalized in [11, 12]. In this framework, we show the existence of a trajectory attractor.

We recall that the usual Cahn-Hilliard equation is characterized by the chemical potential $\mu=-\Delta \varphi+F^{\prime}(\varphi)$. However, this equation has a phenomenological nature. On the contrary, the nonlocal one can be rigorously justified, viewing the standard Cahn-Hilliard equation as its approximation (see $[\mathbf{2 4 , 2 5}$, cf. also [14] and references therein).

The standard (local) system with a singular potential has been analyzed in $[\mathbf{1}, \mathbf{2}, \mathbf{9}]$ (for regular potentials see, e.g., $[22,23,35,37]$ and references therein). Most of the results known for the Navier-Stokes equations essentially hold for the (local) Cahn-Hilliard-Navier-Stokes system as well. On the contrary, in the nonlocal case, due to the weaker smoothness of $\varphi$, proving uniqueness and/or getting higher-order estimates seem a non-trivial task even in dimension two (see $[\mathbf{1 4}, \mathbf{2 1}]$ ). A further interesting and challenging issue is to analyze the sharp interface limit of the nonlocal system (see [3] for a rigorous result in the local case). It is worth mentioning that a nonlocal system for liquid-vapour phase transition has been proposed and analyzed in [33] (see also [27]).

We conclude by observing that the technique we use in $2 \mathrm{D}$ can be easily adapted to show that the 3D (convective) Cahn-Hilliard equation with a singular potential has a connected global (strong) attractor (for regular potentials see [21] and references therein, cf. also $[4, \mathbf{1 7}]$ for results on the local case). To our knowledge this is the first result on the existence of a global attractor for a nonlocal Cahn-Hilliard equation with singular potential.

The plan goes as follows. In the next section, we introduce the weak formulation of our problem. Then we state the existence theorem whose proof is given in Section 3. Section 4 is devoted to the global attractor in 2D, while Section 5 is concerned with the existence of the trajectory attractor whose structural and attraction properties are discussed in Section 6.

\section{Weak solutions and existence theorem}

Let us set $H:=L^{2}(\Omega)$ and $V:=H^{1}(\Omega)$. For every $f \in V^{\prime}$ we denote by $\bar{f}$ the average of $f$ over $\Omega$, i.e.,

$$
\bar{f}:=\frac{1}{|\Omega|}\langle f, 1\rangle .
$$

Here $|\Omega|$ stands for the Lebesgue measure of $\Omega$.

Then we introduce the spaces

$$
V_{0}:=\{v \in V: \bar{v}=0\}, \quad V_{0}^{\prime}:=\left\{f \in V^{\prime}: \bar{f}=0\right\},
$$


and the operator $A: V \rightarrow V^{\prime}, A \in \mathcal{L}\left(V, V^{\prime}\right)$ defined by

$$
\langle A u, v\rangle:=\int_{\Omega} \nabla u \cdot \nabla v \quad \forall u, v \in V .
$$

We recall that $A$ maps $V$ onto $V_{0}^{\prime}$ and the restriction of $A$ to $V_{0}$ maps $V_{0}$ onto $V_{0}^{\prime}$ isomorphically. Let us denote by $\mathcal{N}: V_{0}^{\prime} \rightarrow V_{0}$ the inverse map defined by

$$
A \mathcal{N} f=f, \quad \forall f \in V_{0}^{\prime} \quad \text { and } \quad \mathcal{N} A u=u, \quad \forall u \in V_{0} .
$$

As is well known, for every $f \in V_{0}^{\prime}, \mathcal{N} f$ is the unique solution with zero mean value of the Neumann problem

$$
\left\{\begin{array}{lr}
-\Delta u=f, \quad \text { in } \Omega \\
\frac{\partial u}{\partial n}=0, \quad \text { on } \partial \Omega .
\end{array}\right.
$$

Furthermore, the following relations hold

$$
\begin{aligned}
& \langle A u, \mathcal{N} f\rangle=\langle f, u\rangle, \quad \forall u \in V, \quad \forall f \in V_{0}^{\prime}, \\
& \langle f, \mathcal{N} g\rangle=\langle g, \mathcal{N} f\rangle=\int_{\Omega} \nabla(\mathcal{N} f) \cdot \nabla(\mathcal{N} g), \quad \forall f, g \in V_{0}^{\prime} .
\end{aligned}
$$

We also consider the standard Hilbert spaces for the Navier-Stokes equations (see, e.g., $[36])$

$$
G_{d i v}:={\overline{\left\{u \in C_{0}^{\infty}(\Omega)^{d}: \operatorname{div}(u)=0\right\}^{L}}}^{L^{2}(\Omega)^{d}}, \quad V_{d i v}:=\left\{u \in H_{0}^{1}(\Omega)^{d}: \operatorname{div}(u)=0\right\} .
$$

We denote by $\|\cdot\|$ and $(\cdot, \cdot)$ the norm and the scalar product on both $H$ and $G_{d i v}$, respectively. We recall that $V_{d i v}$ is endowed with the scalar product

$$
(u, v)_{V_{d i v}}=(\nabla u, \nabla v), \quad \forall u, v \in V_{d i v} .
$$

We shall also use the definition of the Stokes operator $S$ with no-slip boundary condition. More precisely, $S: D(S) \subset G_{d i v} \rightarrow G_{d i v}$ is defined as $S:=-P \Delta$ with domain $D(S)=H^{2}(\Omega)^{d} \cap V_{d i v}$, where $P: L^{2}(\Omega)^{d} \rightarrow G_{d i v}$ is the Leray projector. Notice that we have

$$
(S u, v)=(u, v)_{V_{d i v}}=(\nabla u, \nabla v), \quad \forall u \in D(S), \quad \forall v \in V_{d i v}
$$

and $S^{-1}: G_{d i v} \rightarrow G_{d i v}$ is a self-adjoint compact operator in $G_{d i v}$. Thus, according with classical spectral theorems, it possesses a sequence $\left\{\lambda_{j}\right\}$ with $0<\lambda_{1} \leq \lambda_{2} \leq$ $\cdots$ and $\lambda_{j} \rightarrow \infty$, and a family $\left\{w_{j}\right\} \subset D(S)$ of eigenfunctions which is orthonormal in $G_{d i v}$. It is also convenient to recall that the trilinear form $b$ which appears in the weak formulation of the Navier-Stokes equations is defined as follows

$$
b(u, v, w)=\int_{\Omega}(u \cdot \nabla) v \cdot w, \quad \forall u, v, w \in V_{d i v} .
$$

We suppose that the potential $F$ can be written in the following form

$$
F=F_{1}+F_{2},
$$

where $F_{1} \in C^{(2+2 q)}(-1,1)$, with $q$ a fixed positive integer, and $F_{2} \in C^{2}([-1,1])$. 
We can now list the assumptions on the kernel $J$, on the viscosity $\nu$, on $F_{1}, F_{2}$ and on the forcing term $h$.

(A1): $J \in W^{1,1}\left(\mathbb{R}^{d}\right), \quad J(x)=J(-x), \quad a(x):=\int_{\Omega} J(x-y) d y \geq 0, \quad$ a.e. $x \in$ $\Omega$.

(A2): The function $\nu$ is locally Lipschitz on $\mathbb{R}$ and there exist $\nu_{1}, \nu_{2}>0$ such that

$$
\nu_{1} \leq \nu(s) \leq \nu_{2}, \quad \forall s \in \mathbb{R} .
$$

(A3): There exist $c_{1}>0$ and $\epsilon_{0}>0$ such that

$$
F_{1}^{(2+2 q)}(s) \geq c_{1}, \quad \forall s \in\left(-1,-1+\epsilon_{0}\right] \cup\left[1-\epsilon_{0}, 1\right) .
$$

(A4): There exists $\epsilon_{0}>0$ such that, for each $k=0,1, \cdots, 2+2 q$ and each

$$
\begin{aligned}
& j=0,1, \cdots, q, \\
& \quad F_{1}^{(k)}(s) \geq 0, \quad \forall s \in\left[1-\epsilon_{0}, 1\right), \\
& F_{1}^{(2 j+2)}(s) \geq 0, \quad F_{1}^{(2 j+1)}(s) \leq 0, \quad \forall s \in\left(-1,-1+\epsilon_{0}\right] .
\end{aligned}
$$

(A5): There exists $\epsilon_{0}>0$ such that $F_{1}^{(2+2 q)}$ is non-decreasing in $\left[1-\epsilon_{0}, 1\right)$ and non-increasing in $\left(-1,-1+\epsilon_{0}\right]$.

(A6): There exist $\alpha, \beta \in \mathbb{R}$ with $\alpha+\beta>-\min _{[-1,1]} F_{2}^{\prime \prime}$ such that

$$
F_{1}^{\prime \prime}(s) \geq \alpha, \quad \forall s \in(-1,1), \quad a(x) \geq \beta, \quad \text { a.e. } x \in \Omega .
$$

(A7): $\lim _{s \rightarrow \pm 1} F_{1}^{\prime}(s)= \pm \infty$.

(A8): $h \in L^{2}\left(0, T ; V_{d i v}^{\prime}\right)$ for all $T>0$.

REMARK 1. Assumptions (A3)-(A7) are satisfied in the case of the physically relevant logarithmic double-well potential (1.7) for any fixed positive integer $q$. In particular, setting

$$
F_{1}(s)=\frac{\theta}{2}((1+s) \log (1+s)+(1-s) \log (1-s)), \quad F_{2}(s)=-\frac{\theta_{c}}{2} s^{2},
$$

then (A6) is satisfied if and only if $\beta>\theta_{c}-\theta$. However, note that other reasonable potentials satisfy the above assumptions (e.g., the ones which are unbounded at the endpoints).

REMARK 2. The requirement $a(x) \geq \beta$ a.e $x \in \Omega$ is crucial (see [7, Rem.2.1], cf. also [8]). For example, in the case of the double-well smooth potential $F(s)=$ $\left(s^{2}-1\right)^{2}$, which is usually taken as a fairly good smooth approximation of the singular potential, the existence result in [14] requires the condition $a(x) \geq \beta$ with $\beta>4$.

The notion of weak solution to problem (1.1)-(1.6) is given by

Definition 1. Let $u_{0} \in G_{d i v}, \varphi_{0} \in H$ with $F\left(\varphi_{0}\right) \in L^{1}(\Omega)$ and $0<T<+\infty$ be given. A couple $[u, \varphi]$ is a weak solution to (1.1)-(1.6) on $[0, T]$ corresponding to $\left[u_{0}, \varphi_{0}\right]$ if 
- $u, \varphi$ and $\mu$ satisfy

$$
\begin{aligned}
& u \in L^{\infty}\left(0, T ; G_{d i v}\right) \cap L^{2}\left(0, T ; V_{d i v}\right), \\
& u_{t} \in L^{4 / 3}\left(0, T ; V_{d i v}^{\prime}\right), \quad \text { if } d=3, \\
& u_{t} \in L^{2}\left(0, T ; V_{d i v}^{\prime}\right), \quad \text { if } d=2, \\
& \varphi \in L^{\infty}(0, T ; H) \cap L^{2}(0, T ; V), \\
& \varphi_{t} \in L^{2}\left(0, T ; V^{\prime}\right), \\
& \mu=a \varphi-J * \varphi+F^{\prime}(\varphi) \in L^{2}(0, T ; V),
\end{aligned}
$$

and

$$
\varphi \in L^{\infty}(Q), \quad|\varphi(x, t)|<1 \quad \text { a.e. }(x, t) \in Q:=\Omega \times(0, T) ;
$$

- for every $\psi \in V$, every $v \in V_{\text {div }}$ and for almost any $t \in(0, T)$ we have

$$
\begin{aligned}
& \left\langle\varphi_{t}, \psi\right\rangle+(\nabla \mu, \nabla \psi)=(u, \varphi \nabla \psi), \\
& \left\langle u_{t}, v\right\rangle+(2 \nu(\varphi) D u, D v)+b(u, u, v)=-(\varphi \nabla \mu, v)+\langle h, v\rangle ;
\end{aligned}
$$

- the initial conditions $u(0)=u_{0}, \varphi(0)=\varphi_{0}$ hold.

Remark 3. Note that (2.3)-(2.7) imply $u \in C_{w}\left([0, T] ; G_{d i v}\right)\left(u \in C\left([0, T] ; G_{d i v}\right)\right.$ if $d=2)$ and $\varphi \in C([0, T] ; H)$, for all $T>0$. Therefore, the initial conditions $u(0)=u_{0}$ and $\varphi(0)=\varphi_{0}$ make sense.

TheOREm 1. Assume that (A1)-(A8) are satisfied for some fixed positive integer $q$. Let $u_{0} \in G_{d i v}, \varphi_{0} \in L^{\infty}(\Omega)$ such that $F\left(\varphi_{0}\right) \in L^{1}(\Omega)$. In addition, assume that $\left|\overline{\varphi_{0}}\right|<1$. Then, for every $T>0$ there exists a weak solution $z:=[u, \varphi]$ to (1.1)-(1.6) on $[0, T]$ corresponding to $\left[u_{0}, \varphi_{0}\right]$ such that $\bar{\varphi}(t)=\overline{\varphi_{0}}$ for all $t \in[0, T]$ and

$$
\varphi \in L^{\infty}\left(0, T ; L^{2+2 q}(\Omega)\right)
$$

Furthermore, setting

$$
\mathcal{E}(u(t), \varphi(t))=\frac{1}{2}\|u(t)\|^{2}+\frac{1}{4} \int_{\Omega} \int_{\Omega} J(x-y)(\varphi(x, t)-\varphi(y, t))^{2} d x d y+\int_{\Omega} F(\varphi(t)),
$$

the following energy inequality holds

$$
\begin{aligned}
& \mathcal{E}(u(t), \varphi(t))+\int_{s}^{t}\left(2\|\sqrt{\nu(\varphi)} D u(\tau)\|^{2}+\|\nabla \mu(\tau)\|^{2}\right) d \tau \\
& \leq \mathcal{E}(u(s), \varphi(s))+\int_{s}^{t}\langle h(\tau), u(\tau)\rangle d \tau,
\end{aligned}
$$

for all $t \geq s$ and for a.a. $s \in(0, \infty)$, including $s=0$. If $d=2$, the weak solution $z:=[u, \varphi]$ satisfies

$$
\frac{d}{d t} \mathcal{E}(u, \varphi)+2\|\sqrt{\nu(\varphi)} D u\|^{2}+\|\nabla \mu\|^{2}=\langle h, u\rangle,
$$

i.e., equality holds in (2.13) for every $t \geq 0$. 
On account of [21, Corollary 1], the argument used to prove Theorem 1 also yields an existence result for the convective nonlocal Cahn-Hilliard equation with a given velocity field. Note that, in this case, the energy identity holds in 3D as well. In addition, uniqueness goes as in [21, Proposition 5]. Thus we can summarize the results in the following

Proposition 1. Assume that (A1) and (A3)-(A7) are satisfied for some fixed positive integer $q$. Let $u \in L_{l o c}^{2}\left([0, \infty) ; V_{\text {div }} \cap L^{\infty}(\Omega)^{d}\right)$ be given and let $\varphi_{0} \in L^{\infty}(\Omega)$ such that $F\left(\varphi_{0}\right) \in L^{1}(\Omega)$. In addition, suppose that $\left|\overline{\varphi_{0}}\right|<1$. Then, for every $T>0$, there exists a unique $\varphi \in L^{2}(0, T ; V) \cap H^{1}\left(0, T ; V^{\prime}\right)$ which fulfills (2.9) and (2.12), solves $(2.10)$ on $[0, T]$ with $\mu$ given by $(2.8)$ and initial condition $\varphi(0)=\varphi_{0}$. In addition, for all $t \geq 0$, we have $(\varphi(t), 1)=\left(\varphi_{0}, 1\right)$ and the following energy identity holds

$\frac{d}{d t}\left(\frac{1}{4} \int_{\Omega} \int_{\Omega} J(x-y)(\varphi(x, t)-\varphi(y, t))^{2} d x d y+\int_{\Omega} F(\varphi(t))\right)+\|\nabla \mu\|^{2}=(u \varphi, \nabla \mu)$.

REMARK 4. Note that, thanks to (2.6), (2.8) and (2.13), we have that

$$
F^{\prime}(\varphi) \in L^{2}(0, T ; V), \quad F(\varphi) \in L^{\infty}\left(0, T ; L^{1}(\Omega)\right), \quad \forall T>0 .
$$

Remark 5. Assumptions (A3)-(A6) ensure that, thanks to Lemma 1 and Lemma 2, property (2.12) holds for some fixed $q \geq 1$. Indeed, such assumptions allow us to obtain some estimates for the approximating (regular) potential $F_{\epsilon}$ which are crucial in the approximation argument of the proof of Theorem 1 (see (3.9) and (3.11) below). Actually, Theorem 1 states that for each $q \geq 1$ there exists a solution satisfying (2.12). Notice that, since the $L^{\infty}\left(0, T ; L^{\infty}(\Omega)\right)$-regularity of $\varphi$ is not guaranteed (since $\varphi$ might not be measurable with values in $L^{\infty}(\Omega)$ ), we cannot rely on such a regularity in order to choose some fixed $q$ (e.g., $q=1$ ) for $F_{\epsilon}$ (cf. (3.10) below). Furthermore, (2.12) does not follow from (2.9). Indeed, recall that $L^{\infty}\left(0, T ; L^{\infty}(\Omega)\right) \subset L^{\infty}(Q)$ with strict inclusion.

\section{Proof of Theorem 1}

We consider the following approximate problem $P_{\epsilon}$ : find a weak solution $\left[u_{\epsilon}, \varphi_{\epsilon}\right]$ to

$$
\begin{aligned}
& \varphi_{\epsilon}^{\prime}+u_{\epsilon} \cdot \nabla \varphi_{\epsilon}=\Delta \mu_{\epsilon}, \\
& u_{\epsilon}^{\prime}-\operatorname{div}\left(\nu\left(\varphi_{\epsilon}\right) 2 D u_{\epsilon}\right)+\left(u_{\epsilon} \cdot \nabla\right) u_{\epsilon}+\nabla \pi_{\epsilon}=\mu_{\epsilon} \nabla \varphi_{\epsilon}+h, \\
& \mu_{\epsilon}=a \varphi_{\epsilon}-J * \varphi_{\epsilon}+F_{\epsilon}^{\prime}\left(\varphi_{\epsilon}\right), \\
& \operatorname{div}\left(u_{\epsilon}\right)=0, \\
& \frac{\partial \mu_{\epsilon}}{\partial n}=0, \quad u_{\epsilon}=0, \quad \text { on } \partial \Omega, \\
& u_{\epsilon}(0)=u_{0}, \quad \varphi_{\epsilon}(0)=\varphi_{0}, \quad \text { in } \Omega .
\end{aligned}
$$


Problem $P_{\epsilon}$ is obtained from (1.1)-(1.6) by replacing the singular potential $F$ with the smooth potential

$$
F_{\epsilon}=F_{1 \epsilon}+\bar{F}_{2}
$$

where $F_{1 \epsilon}$ is defined by

$$
F_{1 \epsilon}^{(2+2 q)}(s)=\left\{\begin{array}{l}
F_{1}^{(2+2 q)}(1-\epsilon), \quad s \geq 1-\epsilon \\
F_{1}^{(2+2 q)}(s), \quad|s| \leq 1-\epsilon \\
F_{1}^{(2+2 q)}(-1+\epsilon), \quad s \leq-1+\epsilon
\end{array}\right.
$$

and $F_{1 \epsilon}(0)=F_{1}(0), F_{1 \epsilon}^{\prime}(0)=F_{1}^{\prime}(0), \ldots F_{1 \epsilon}^{(1+2 q)}(0)=F_{1}^{(1+2 q)}(0)$, while $\bar{F}_{2}$ is a $C^{2}(\mathbb{R})$-extension of $F_{2}$ on $\mathbb{R}$ with polynomial growth satisfying

$$
\bar{F}_{2}(s) \geq \min _{[-1,1]} F_{2}-1, \quad \bar{F}_{2}^{\prime \prime}(s) \geq \min _{[-1,1]} F_{2}^{\prime \prime}, \quad \forall s \in \mathbb{R} .
$$

The following elementary lemmas are basic to obtain uniform (w.r.t. $\epsilon$ ) estimates for a weak solution to the approximate problem.

Lemma 1. Suppose that (A3) and (A4) hold. Then, there exist $c_{q}, d_{q}>0$, which depend on $q$ but are independent of $\epsilon$, and $\epsilon_{0}>0$ such that

$$
F_{\epsilon}(s) \geq c_{q}|s|^{2+2 q}-d_{q}, \quad \forall s \in \mathbb{R}, \quad \forall \epsilon \in\left(0, \epsilon_{0}\right] .
$$

Proof. By integrating (3.7) we get

$$
F_{1 \epsilon}(s)=\left\{\begin{array}{l}
\sum_{k=0}^{2+2 q} \frac{1}{k !} F_{1}^{(k)}(1-\epsilon)[s-(1-\epsilon)]^{k}, \quad s \geq 1-\epsilon \\
F_{1}(s), \quad|s| \leq 1-\epsilon \\
\sum_{k=0}^{2+2 q} \frac{1}{k !} F_{1}^{(k)}(-1+\epsilon)[s-(-1+\epsilon)]^{k}, \quad s \leq-1+\epsilon .
\end{array}\right.
$$

Due to (A4) we have, for $\epsilon$ small enough,

$$
F_{1 \epsilon}(s) \geq \frac{1}{(2+2 q) !} F_{1}^{(2+2 q)}(1-\epsilon)[s-(1-\epsilon)]^{2+2 q}, \quad \forall s \geq 1-\epsilon,
$$

so that, in particular,

$$
F_{1 \epsilon}(s) \geq \frac{1}{(2+2 q) !} F_{1}^{(2+2 q)}(1-\epsilon)(s-1)^{2+2 q}, \quad \forall s \geq 1,
$$

and (A3) implies that (for $\epsilon$ small enough)

$$
F_{1 \epsilon}(s) \geq 2 c_{q}(s-1)^{2+2 q} \geq c_{q} s^{2+2 q}-d_{q}, \quad \forall s \geq 1,
$$

where $c_{q}=c_{1} / 2(2+2 q)$ ! and $d_{q}$ is another constant depending only on $q$. Furthermore, we have $F_{1 \epsilon}(s)=F_{1}(s) \geq 0 \geq c_{q} s^{2+2 q}-d_{q}$ for $0 \leq s \leq 1-\epsilon$, provided we choose $d_{q} \geq c_{q}$, while for $1-\epsilon \leq s \leq 1$ we have $F_{1 \epsilon} \geq 2 c_{q}[s-(1-\epsilon)]^{2+2 q} \geq$ $0 \geq c_{q} s^{2+2 q}-d_{q}$. Summing up, we deduce that there exists $\epsilon_{0}>0$ such that $F_{1 \epsilon}(s) \geq c_{q} s^{2+2 q}-d_{q}$, for all $s \geq 0$ and for all $\epsilon \in\left(0, \epsilon_{0}\right]$. By using (3.8) we also get (3.9) for $s \geq 0$. Similarly we obtain (3.9) for $s \leq 0$. 
Lemma 2. Suppose (A4) and (A6) hold. Then, setting $c_{0}:=\alpha+\beta+\min _{[-1,1]} F_{2}^{\prime \prime}>$ 0 , there exists $\epsilon_{1}>0$ such that

$$
F_{\epsilon}^{\prime \prime}(s)+a(x) \geq c_{0}, \quad \forall s \in \mathbb{R}, \quad \text { a.e. } x \in \Omega, \quad \forall \epsilon \in\left(0, \epsilon_{1}\right] .
$$

Proof. From (3.10) we have

$$
F_{1 \epsilon}^{\prime \prime}(s)= \begin{cases}\sum_{k=0}^{2 q} \frac{1}{k !} F_{1}^{(k+2)}(1-\epsilon)[s-(1-\epsilon)]^{k}, & s \geq 1-\epsilon \\ F_{1}^{\prime \prime}(s), \quad|s| \leq 1-\epsilon & \\ \sum_{k=0}^{2 q} \frac{1}{k !} F_{1}^{(k)}(-1+\epsilon)[s-(-1+\epsilon)]^{k}, & s \leq-1+\epsilon .\end{cases}
$$

Assumption (A4) implies that for $\epsilon$ small enough $F_{1 \epsilon}^{\prime \prime}(s) \geq F_{1}^{\prime \prime}(1-\epsilon)$ for $s \geq 1-\epsilon$ and $F_{1 \epsilon}^{\prime \prime}(s) \geq F_{1}^{\prime \prime}(-1+\epsilon)$ for $s \leq-1+\epsilon$. Since $F_{1 \epsilon}^{\prime \prime}(s)=F_{1}^{\prime \prime}(s)$ for $|s| \leq 1-\epsilon$, (A6) implies that there exists $\epsilon_{1}>0$ such that

$$
F_{1 \epsilon}^{\prime \prime}(s) \geq \alpha, \quad \forall s \in \mathbb{R}, \quad \forall \epsilon \in\left(0, \epsilon_{1}\right] .
$$

This estimate together with (3.8) and (A6) imply (3.11).

Due to the existence result proved in [14], for every $T>0$, Problem $P_{\epsilon}$ admits a weak solution $z_{\epsilon}:=\left[u_{\epsilon}, \varphi_{\epsilon}\right]$ such that

$$
\begin{aligned}
& u_{\epsilon} \in L^{\infty}\left(0, T ; G_{d i v}\right) \cap L^{2}\left(0, T ; V_{d i v}\right), \\
& u_{\epsilon}^{\prime} \in L^{4 / 3}\left(0, T ; V_{d i v}^{\prime}\right), \quad \text { if } \quad d=3, \\
& u_{\epsilon}^{\prime} \in L^{2}\left(0, T ; V_{d i v}^{\prime}\right), \quad \text { if } \quad d=2, \\
& \varphi_{\epsilon} \in L^{\infty}\left(0, T ; L^{2+2 q}(\Omega)\right) \cap L^{2}(0, T ; V), \\
& \varphi_{\epsilon}^{\prime} \in L^{2}\left(0, T ; V^{\prime}\right), \\
& \mu_{\epsilon} \in L^{2}(0, T ; V) .
\end{aligned}
$$

Indeed, it is immediate to check that all the assumptions of $[\mathbf{1 4}$, Theorem 1] and of [14, Corollary 1] are satisfied for Problem $P_{\epsilon}$. In particular, we use Lemma 1, Lemma 2 and the fact that, due to the definition of $F_{1 \epsilon}$ and to the polynomial growth assumption on $\bar{F}_{2}$, assumption (H5) of [14, Theorem 1] is trivially satisfied for each $\epsilon>0$ (with some constants depending on $\epsilon$ ).

Furthermore, according to [14, Theorem 1] and using (A2), the approximate solution $z_{\epsilon}:=\left[u_{\epsilon}, \varphi_{\epsilon}\right]$ satisfies the following energy inequality

$$
\begin{aligned}
& \frac{1}{2}\left\|u_{\epsilon}(t)\right\|^{2}+\frac{1}{4} \int_{\Omega} \int_{\Omega} J(x-y)\left(\varphi_{\epsilon}(x, t)-\varphi_{\epsilon}(y, t)\right)^{2} d x d y+\int_{\Omega} F_{\epsilon}\left(\varphi_{\epsilon}(t)\right) \\
& +\int_{0}^{t}\left(\nu_{1}\left\|\nabla u_{\epsilon}\right\|^{2}+\left\|\nabla \mu_{\epsilon}\right\|^{2}\right) d \tau \leq \frac{1}{2}\left\|u_{0}\right\|^{2} \\
& +\frac{1}{4} \int_{\Omega} \int_{\Omega} J(x-y)\left(\varphi_{0}(x)-\varphi_{0}(y)\right)^{2} d x d y \\
& +\int_{\Omega} F_{\epsilon}\left(\varphi_{0}\right)+\int_{0}^{t}\left\langle h, u_{\epsilon}\right\rangle d \tau, \quad \forall t \in[0, T]
\end{aligned}
$$


From (A5) it is easy to see (cf. (3.33) and (3.34) below) that there exists $\epsilon_{1}>0$ such that

$$
F_{1 \epsilon}(s) \leq F_{1}(s), \quad \forall s \in(-1,1), \quad \forall \epsilon \in\left(0, \epsilon_{1}\right] .
$$

Therefore, using the assumptions on $\varphi_{0}, u_{0}$ and Lemma 1, from (3.20) we get the following estimates

$$
\begin{aligned}
& \left\|u_{\epsilon}\right\|_{L^{\infty}\left(0, T ; G_{d i v}\right) \cap L^{2}\left(0, T ; V_{d i v}\right)} \leq c, \\
& \left\|\varphi_{\epsilon}\right\|_{L^{\infty}\left(0, T ; L^{2+2 q}(\Omega)\right)} \leq c, \\
& \left\|\nabla \mu_{\epsilon}\right\|_{L^{2}(0, T ; H)} \leq c .
\end{aligned}
$$

Henceforth $c$ will denote a positive constant which depends on the initial data (i.e., on $\left.\left\|u_{0}\right\|,\left\|\varphi_{0}\right\|, \int_{\Omega} F\left(\varphi_{0}\right)\right)$ and on $\|h\|_{L^{2}\left(0, T ; V_{d i v}^{\prime}\right.}, F, \Omega, J, \nu_{1}$, but is independent of $\epsilon$.

We then take the gradient of (3.3) and multiply the resulting identity by $\nabla \varphi_{\epsilon}$ in $L^{2}(\Omega)$. Arguing as in [14, Proof of Theorem 1] and using (3.11), we get

$$
\left\|\nabla \mu_{\epsilon}\right\|^{2} \geq \frac{c_{0}^{2}}{4}\left\|\nabla \varphi_{\epsilon}\right\|^{2}-k\left\|\varphi_{\epsilon}\right\|^{2},
$$

with $k=2\|\nabla J\|_{L^{1}}^{2}$. This last estimate together with (3.23) and (3.24) yield

$$
\left\|\varphi_{\epsilon}\right\|_{L^{2}(0, T ; V)} \leq c .
$$

As far as the bounds on the time derivatives $\left\{u_{\epsilon}^{\prime}\right\}$ and $\left\{\varphi_{\epsilon}^{\prime}\right\}$ are concerned, on account of (3.1) and (3.2), arguing by comparison as in [14, Proof of Theorem 1], one gets

$$
\begin{aligned}
& \left\|\varphi_{\epsilon}^{\prime}\right\|_{L^{2}\left(0, T ; V^{\prime}\right)} \leq c, \\
& \left\|u_{\epsilon}^{\prime}\right\|_{L^{2}\left(0, T ; V_{d i v}^{\prime}\right)} \leq c, \quad d=2 \\
& \left\|u_{\epsilon}^{\prime}\right\|_{L^{4 / 3}\left(0, T ; V_{d i v}^{\prime}\right)} \leq c, \quad d=3 .
\end{aligned}
$$

In order to obtain an estimate for $\left\{\mu_{\epsilon}\right\}$ we need to control the sequence of averages $\left\{\overline{\mu_{\epsilon}}\right\}$. To this aim observe that equation (3.1) can be written in abstract form as follows

$$
\varphi_{\epsilon}^{\prime}+u_{\epsilon} \cdot \nabla \varphi_{\epsilon}=-A \mu_{\epsilon} \quad \text { in } V^{\prime} .
$$

Let us test (3.29) by $\mathcal{N}\left(F_{\epsilon}^{\prime}\left(\varphi_{\epsilon}\right)-\overline{F_{\epsilon}^{\prime}\left(\varphi_{\epsilon}\right)}\right)$ to get

$$
\begin{aligned}
& \left\langle F_{\epsilon}^{\prime}\left(\varphi_{\epsilon}\right)-\overline{F_{\epsilon}^{\prime}\left(\varphi_{\epsilon}\right)}, \mathcal{N} \varphi_{\epsilon}^{\prime}\right\rangle+\left\langle\mathcal{N}\left(u_{\epsilon} \cdot \nabla \varphi_{\epsilon}\right), F_{\epsilon}^{\prime}\left(\varphi_{\epsilon}\right)-\overline{F_{\epsilon}^{\prime}\left(\varphi_{\epsilon}\right)}\right\rangle \\
& =-\left\langle\mu_{\epsilon}, F_{\epsilon}^{\prime}\left(\varphi_{\epsilon}\right)-\overline{F_{\epsilon}^{\prime}\left(\varphi_{\epsilon}\right)}\right\rangle .
\end{aligned}
$$

Recall that $\overline{u_{\epsilon} \cdot \nabla \varphi_{\epsilon}}=0$. On the other hand, we have

$$
\begin{aligned}
& \left\langle\mu_{\epsilon}, F_{\epsilon}^{\prime}\left(\varphi_{\epsilon}\right)-\overline{F_{\epsilon}^{\prime}\left(\varphi_{\epsilon}\right)}\right\rangle= \\
& \left\langle a \varphi_{\epsilon}-J * \varphi_{\epsilon}+F_{\epsilon}^{\prime}\left(\varphi_{\epsilon}\right)-\overline{F_{\epsilon}^{\prime}\left(\varphi_{\epsilon}\right)}, F_{\epsilon}^{\prime}\left(\varphi_{\epsilon}\right)-\overline{F_{\epsilon}^{\prime}\left(\varphi_{\epsilon}\right)}\right\rangle
\end{aligned}
$$




$$
\begin{aligned}
& \geq \frac{1}{2}\left\|F_{\epsilon}^{\prime}\left(\varphi_{\epsilon}\right)-\overline{F_{\epsilon}^{\prime}\left(\varphi_{\epsilon}\right)}\right\|^{2}-\frac{1}{2}\left\|a \varphi_{\epsilon}-J * \varphi_{\epsilon}\right\|^{2} \\
& \geq \frac{1}{2}\left\|F_{\epsilon}^{\prime}\left(\varphi_{\epsilon}\right)-\overline{F_{\epsilon}^{\prime}\left(\varphi_{\epsilon}\right)}\right\|^{2}-C_{J}\left\|\varphi_{\epsilon}\right\|^{2} .
\end{aligned}
$$

Therefore, by means of (3.31) and (3.23), from (3.30) we deduce

$$
\begin{aligned}
& \left\|F_{\epsilon}^{\prime}\left(\varphi_{\epsilon}\right)-\overline{F_{\epsilon}^{\prime}\left(\varphi_{\epsilon}\right)}\right\| \leq c\left(\left\|\mathcal{N} \varphi_{\epsilon}^{\prime}\right\|+\left\|\mathcal{N}\left(u_{\epsilon} \cdot \nabla \varphi_{\epsilon}\right)\right\|+1\right) \\
& \leq c\left(\left\|\varphi_{\epsilon}^{\prime}\right\|_{V_{0}^{\prime}}+\left\|u_{\epsilon} \cdot \nabla \varphi_{\epsilon}\right\|_{V_{0}^{\prime}}+1\right) .
\end{aligned}
$$

Observe now that, due to (A4) and (A5), there holds

$$
\left|F_{1 \epsilon}^{\prime}(s)\right| \leq\left|F_{1}^{\prime}(s)\right|, \quad \forall s \in(-1,1), \quad \forall \epsilon \in\left(0, \epsilon_{1}\right],
$$

for some $\epsilon_{1}>0$. Indeed, for $s \in[1-\epsilon, 1)$ we have

$$
\begin{aligned}
& F_{1}^{\prime}(s)=\sum_{k=0}^{2 q} \frac{1}{k !} F_{1}^{(k+1)}(1-\epsilon)[s-(1-\epsilon)]^{k} \\
& +\frac{1}{(2 q+1) !} F_{1}^{(2 q+2)}(\xi)[s-(1-\epsilon)]^{1+2 q} \\
& \geq \sum_{k=0}^{1+2 q} \frac{1}{k !} F_{1}^{(k+1)}(1-\epsilon)[s-(1-\epsilon)]^{k}=F_{1 \epsilon}^{\prime}(s),
\end{aligned}
$$

for $\epsilon$ small enough, where $\xi \in(1-\epsilon, s)$ and where we have used the fact that, due to $(\mathrm{A} 5), F_{1}^{(2+2 q)}(\xi) \geq F_{1}^{(2+2 q)}(1-\epsilon)$. Arguing similarly, we get $F_{1 \epsilon}^{\prime}(s) \geq F_{1}^{\prime}(s)$ for $s \in(-1,-1+\epsilon]$ and for $\epsilon$ small enough. However, due to (A4) and (A7), for $\epsilon$ small enough we have that $F_{1 \epsilon}^{\prime}(s) \geq F_{1}^{\prime}(1-\epsilon) \geq 0$ for $s \geq 1-\epsilon$ and $F_{1 \epsilon}^{\prime}(s) \leq F_{1}^{\prime}(-1+\epsilon) \leq 0$ for $s \leq-1+\epsilon$. Recalling also that $F_{1 \epsilon}^{\prime}(s)=F_{1}^{\prime}(s)$ for $|s| \leq 1-\epsilon$, we obtain (3.33).

Let $s_{0} \in(-1,1)$ be such that $F^{\prime}\left(s_{0}\right)=0$ (cf. (A7)) and introduce

$$
H(s):=F(s)+\frac{a_{\infty}}{2}\left(s-s_{0}\right)^{2}, \quad H_{\epsilon}(s):=F_{\epsilon}(s)+\frac{a_{\infty}}{2}\left(s-s_{0}\right)^{2},
$$

for every $s \in(-1,1)$ and every $s \in \mathbb{R}$, respectively. We have set $a_{\infty}:=\|a\|_{L^{\infty}(\Omega)}$. Observe that, owing to (3.11), $H_{\epsilon}^{\prime}$ is monotone and (for $\epsilon$ small enough) $H_{\epsilon}^{\prime}\left(s_{0}\right)=$ $F^{\prime}\left(s_{0}\right)=0$. Since $\overline{\varphi_{0}} \in(-1,1)$, we can apply an argument devised by Kenmochi et al. [31] (see also [15]) and deduce the following estimate

$$
\delta\left\|H_{\epsilon}^{\prime}\left(\varphi_{\epsilon}\right)\right\|_{L^{1}(\Omega)} \leq \int_{\Omega}\left(\varphi_{\epsilon}-\overline{\varphi_{0}}\right)\left(H_{\epsilon}^{\prime}\left(\varphi_{\epsilon}\right)-\overline{H_{\epsilon}^{\prime}\left(\varphi_{\epsilon}\right)}\right)+K\left(\overline{\varphi_{0}}\right)
$$

where $\delta$ depends on $\overline{\varphi_{0}}$ and $K\left(\overline{\varphi_{0}}\right)$ depends on $\overline{\varphi_{0}}, F,|\Omega|$ and on $a_{\infty}$. For the reader's convenience let us recall briefly how (3.36) can be deduced. Fix $m_{1}, m_{2} \in$ $(-1,1)$ such that $m_{1} \leq s_{0} \leq m_{2}$ and $m_{1}<\overline{\varphi_{0}}<m_{2}$. Introduce, for a.a. fixed $t \in(0, T)$, the sets

$$
\Omega_{0}:=\left\{m_{1} \leq \varphi_{\epsilon}(x, t) \leq m_{2}\right\}, \quad \Omega_{1}:=\left\{\varphi_{\epsilon}(x, t)<m_{1}\right\}, \quad \Omega_{2}:=\left\{\varphi_{\epsilon}(x, t)>m_{2}\right\} .
$$


Setting $\delta:=\min \left\{\overline{\varphi_{0}}-m_{1}, m_{2}-\overline{\varphi_{0}}\right\}$ and $\delta_{1}:=\max \left\{\overline{\varphi_{0}}-m_{1}, m_{2}-\overline{\varphi_{0}}\right\}$, then for $\epsilon$ small enough we have

$$
\begin{aligned}
& \delta\left\|H_{\epsilon}^{\prime}\left(\varphi_{\epsilon}\right)\right\|_{L^{1}(\Omega)}=\delta \int_{\Omega_{1}}\left|H_{\epsilon}^{\prime}\left(\varphi_{\epsilon}\right)\right|+\delta \int_{\Omega_{2}}\left|H_{\epsilon}^{\prime}\left(\varphi_{\epsilon}\right)\right|+\delta \int_{\Omega_{0}}\left|H_{\epsilon}^{\prime}\left(\varphi_{\epsilon}\right)\right| \\
& \leq \int_{\Omega_{1}}\left(\varphi_{\epsilon}(t)-\overline{\varphi_{0}}\right) H_{\epsilon}^{\prime}\left(\varphi_{\epsilon}\right)+\int_{\Omega_{2}}\left(\varphi_{\epsilon}(t)-\overline{\varphi_{0}}\right) H_{\epsilon}^{\prime}\left(\varphi_{\epsilon}\right)+\delta \int_{\Omega_{0}}\left|H_{\epsilon}^{\prime}\left(\varphi_{\epsilon}\right)\right| \\
& \leq \int_{\Omega}\left(\varphi_{\epsilon}(t)-\overline{\varphi_{0}}\right) H_{\epsilon}^{\prime}\left(\varphi_{\epsilon}\right)+\left(\delta_{1}+\delta\right) \int_{\Omega_{0}}\left|H_{\epsilon}^{\prime}\left(\varphi_{\epsilon}\right)\right| \\
& \leq \int_{\Omega}\left(\varphi_{\epsilon}(t)-\overline{\varphi_{0}}\right) H_{\epsilon}^{\prime}\left(\varphi_{\epsilon}\right)+\left(\delta_{1}+\delta\right) \int_{\Omega_{0}}\left\{\left|F_{1}^{\prime}\left(\varphi_{\epsilon}\right)\right|+\left|F_{2}^{\prime}\left(\varphi_{\epsilon}\right)\right|+a_{\infty}\left|\varphi_{\epsilon}-s_{0}\right|\right\},
\end{aligned}
$$

where we have used (3.33). We therefore get (3.36) with $K\left(\overline{\varphi_{0}}\right)$ given by

$$
K\left(\overline{\varphi_{0}}\right)=\left(\delta_{1}+\delta\right)|\Omega|\left(\max _{\left[m_{1}, m_{2}\right]}\left(\left|F_{1}^{\prime}\right|+\left|F_{2}^{\prime}\right|\right)+a_{\infty} \delta_{2}\right)
$$

with $\delta_{2}:=\max \left\{s_{0}-m_{1}, m_{2}-s_{0}\right\}$. On account of the definition of $H_{\epsilon}$ and recalling (3.32) we obtain

$$
\left\|H_{\epsilon}^{\prime}\left(\varphi_{\epsilon}\right)-\overline{H_{\epsilon}^{\prime}\left(\varphi_{\epsilon}\right)}\right\| \leq c\left(\left\|\varphi_{\epsilon}^{\prime}\right\|_{V_{0}^{\prime}}+\left\|u_{\epsilon} \cdot \nabla \varphi_{\epsilon}\right\|_{V_{0}^{\prime}}+1\right)+a_{\infty}\left\|\varphi_{\epsilon}-\overline{\varphi_{0}}\right\| .
$$

Therefore, by means of (3.36)-(3.37) and using the following bound (cf. (3.22) and (3.23), see [14, Proof of Corollary 1])

$$
\left\|u_{\epsilon} \cdot \nabla \varphi_{\epsilon}\right\|_{L^{2}\left(0, T ; V_{0}^{\prime}\right)} \leq c
$$

we infer that there exists

a constant $L\left(\overline{\varphi_{0}}\right)$ depending on $\overline{\varphi_{0}}$ such that

$$
\left\|F_{\epsilon}^{\prime}\left(\varphi_{\epsilon}\right)\right\|_{L^{2}\left(0, T ; L^{1}(\Omega)\right)} \leq L\left(\overline{\varphi_{0}}\right) .
$$

Since $\int_{\Omega} \mu_{\epsilon}=\int_{\Omega} F_{\epsilon}^{\prime}\left(\varphi_{\epsilon}\right)$, then $\left\|\overline{\mu_{\epsilon}}\right\|_{L^{2}(0, T)} \leq c$. Hence by Poincaré-Wirtinger inequality and (3.24) we get

$$
\left\|\mu_{\epsilon}\right\|_{L^{2}(0, T ; V)} \leq c
$$

Estimates (3.22), (3.23), (3.25)-(3.28), (3.39) and well-known compactness results allow us to deduce that there exist functions $u \in L^{\infty}\left(0, T ; G_{\text {div }}\right) \cap L^{2}\left(0, T ; V_{\text {div }}\right)$, $\varphi \in L^{\infty}\left(0, T ; L^{2+2 q}(\Omega)\right) \cap L^{2}(0, T ; V) \cap H^{1}\left(0, T ; V^{\prime}\right)$, and $\mu \in L^{2}(0, T ; V)$ such that, up to a subsequence, we have

$$
\begin{array}{ll}
u_{\epsilon} \rightarrow u & \text { weakly* in } L^{\infty}\left(0, T ; G_{d i v}\right), \quad \text { weakly in } L^{2}\left(0, T ; V_{d i v}\right), \\
u_{\epsilon} \rightarrow u & \text { strongly in } L^{2}\left(0, T ; G_{d i v}\right), \quad \text { a.e. in } \Omega \times(0, T), \\
u_{\epsilon}^{\prime} \rightarrow u_{t} & \text { weakly in } L^{4 / 3}\left(0, T ; V_{d i v}^{\prime}\right), \quad d=3, \\
u_{\epsilon}^{\prime} \rightarrow u_{t} & \text { weakly in } L^{2}\left(0, T ; V_{d i v}^{\prime}\right), \quad d=2, \\
\varphi_{\epsilon} \rightarrow \varphi & \text { weakly* in } L^{\infty}\left(0, T ; L^{2+2 q}(\Omega)\right), \quad \text { weakly in } L^{2}(0, T ; V), \\
\varphi_{\epsilon} \rightarrow \varphi & \text { strongly in } L^{2}(0, T ; H), \quad \text { a.e. in } \Omega \times(0, T), \\
\varphi_{\epsilon}^{\prime} \rightarrow \varphi_{t} & \text { weakly in } L^{2}\left(0, T ; V^{\prime}\right),
\end{array}
$$




$$
\mu_{\epsilon} \rightarrow \mu \quad \text { weakly in } L^{2}(0, T ; V) .
$$

In order to pass to the limit in the variational formulation for Problem $P_{\epsilon}$ and hence prove that $z=[u, \varphi]$ is a weak solution to the original problem, we need to show that $|\varphi|<1$ a.e. in $Q=\Omega \times(0, T)$. To this aim we adapt an argument devised in [17] (cf. also [19]).

Thus, we introduce the sets

$$
E_{1, \eta}^{\epsilon}:=\left\{(x, t) \in Q: \varphi_{\epsilon}(x, t)>1-\eta\right\}, \quad E_{2, \eta}^{\epsilon}:=\left\{(x, t) \in Q: \varphi_{\epsilon}(x, t)<-1+\eta\right\},
$$

where $\eta \in(0,1)$ is chosen so that $s_{0} \in(-1+\eta, 1-\eta)$ with $s_{0}$ such that $F^{\prime}\left(s_{0}\right)=0$. For $\epsilon$ small enough, recalling that $H_{\epsilon}^{\prime}(s) \geq 0$ for $s \in\left[s_{0}, 1\right)$ and $H_{\epsilon}^{\prime}(s) \leq 0$ for $s \in\left(-1, s_{0}\right]$, we can write

$$
H_{\epsilon}^{\prime}(1-\eta)\left|E_{1, \eta}^{\epsilon}\right|_{d+1} \leq\left\|H_{\epsilon}^{\prime}\left(\varphi_{\epsilon}\right)\right\|_{L^{1}(Q)}, \quad\left|H_{\epsilon}^{\prime}(-1+\eta)\left\|\left.E_{2, \eta}^{\epsilon}\right|_{d+1} \leq\right\| H_{\epsilon}^{\prime}\left(\varphi_{\epsilon}\right) \|_{L^{1}(Q)},\right.
$$

where $|\cdot|_{d+1}$ is the $d+1$-Lebesgue measure in $Q$, and observe that $\left\|H_{\epsilon}^{\prime}\left(\varphi_{\epsilon}\right)\right\|_{L^{1}(Q)} \leq$ $c L\left(\overline{\varphi_{0}}\right)+c$ (cf. (3.23) and (3.38)). Furthermore, as a consequence of the pointwise convergence (3.45) and by using Fatou's lemma, it is easy to see that we have

$$
\left|E_{1, \eta}\right|_{d+1} \leq \liminf _{\epsilon \rightarrow 0}\left|E_{1, \eta}^{\epsilon}\right|_{d+1}, \quad\left|E_{2, \eta}\right|_{d+1} \leq \liminf _{\epsilon \rightarrow 0}\left|E_{2, \eta}^{\epsilon}\right|_{d+1},
$$

where

$$
E_{1, \eta}:=\{(x, t) \in Q: \varphi(x, t)>1-\eta\}, \quad E_{2, \eta}:=\{(x, t) \in Q: \varphi(x, t)<-1+\eta\} .
$$

Hence, due to the pointwise convergence $H_{\epsilon}^{\prime}(s) \rightarrow H^{\prime}(s)$, for every $s \in(-1,1)$, we get from (3.48) and (3.49)

$$
\left|E_{1, \eta}\right|_{d+1} \leq \frac{c L\left(\overline{\varphi_{0}}\right)+c}{H^{\prime}(1-\eta)}, \quad\left|E_{2, \eta}\right|_{d+1} \leq \frac{c L\left(\overline{\varphi_{0}}\right)+c}{\left|H^{\prime}(-1+\eta)\right|} .
$$

Letting $\eta \rightarrow 0$ and using (A7) we obtain $|\{(x, t) \in Q:|\varphi(x, t)| \geq 1\}|=0$ and therefore $|\varphi(x, t)|<1$ for a.e. $(x, t) \in Q$.

This bound, the pointwise convergence (3.45) in $Q$ and the fact that $F_{\epsilon}^{\prime} \rightarrow F^{\prime}$ uniformly on every compact interval included in $(-1,1)$, entail that

$$
F_{\epsilon}^{\prime}\left(\varphi_{\epsilon}\right) \rightarrow F^{\prime}(\varphi) \text { a.e. in } Q .
$$

Convergences (3.40)-(3.47) and (3.51) allow us, by a standard argument, to pass to the limit in the variational formulation of Problem $P_{\epsilon}$ and hence to prove that $z=[u, \varphi]$ is a weak solution to (1.1)-(1.6).

Let us now establish the energy inequality (2.13). Let us first show that (2.13) holds for $s=0$ and $t>0$. Indeed, the energy inequality satisfied by the approximate solution $z_{\epsilon}=\left[u_{\epsilon}, \varphi_{\epsilon}\right]$ can be written as follows

$$
\begin{aligned}
& \frac{1}{2}\left\|u_{\epsilon}(t)\right\|^{2}+\frac{1}{2}\left\|\sqrt{a} \varphi_{\epsilon}(t)\right\|^{2} \\
& -\frac{1}{2}\left(\varphi_{\epsilon}(t), J * \varphi_{\epsilon}(t)\right)+\int_{\Omega} F_{\epsilon}\left(\varphi_{\epsilon}(t)\right)
\end{aligned}
$$




$$
\begin{aligned}
& +\int_{0}^{t}\left(2\left\|\sqrt{\nu\left(\varphi_{\epsilon}\right)} D u_{\epsilon}\right\|^{2}+\left\|\nabla \mu_{\epsilon}\right\|^{2}\right) d \tau \\
& \leq \frac{1}{2}\left\|u_{0}\right\|^{2}+\frac{1}{2}\left\|\sqrt{a} \varphi_{0}\right\|^{2}-\frac{1}{2}\left(\varphi_{0}, J * \varphi_{0}\right) \\
& +\int_{\Omega} F_{\epsilon}\left(\varphi_{0}\right)+\int_{0}^{t}\left\langle h, u_{\epsilon}\right\rangle d \tau, \quad \forall t>0 .
\end{aligned}
$$

We now use the strong convergences (3.41) and (3.45), the weak convergences (3.40) and (3.47), the bound (3.21) for the approximate potential $F_{1 \epsilon}$, the fact that $F_{\epsilon}\left(\varphi_{\epsilon}(t)\right) \rightarrow F(\varphi(t))$ a.e. in $\Omega$ and for a.e. $t \in(0, T)$ (see (3.51)) and Fatou's lemma. Observe that, as a consequence of the uniform bound $\left\|\sqrt{\nu\left(\varphi_{\epsilon}\right)}\right\|_{\infty} \leq \sqrt{\nu_{2}}$ (cf. assumption (A2)), of the strong convergence $\sqrt{\nu\left(\varphi_{\epsilon}\right)} \rightarrow \sqrt{\nu(\varphi)}$ in $L^{2}(0, T ; H)$ and of the weak convergence (3.40), we have (see, e.g., [14, Lemma1])

$$
\sqrt{\nu\left(\varphi_{\epsilon}\right)} D u_{\epsilon} \rightarrow \sqrt{\nu(\varphi)} D u, \quad \text { weakly in } L^{2}(0, T ; H) .
$$

By letting $\epsilon \rightarrow 0$, from (3.52) we infer that (2.13) holds for almost every $t>$ 0 . Furthermore, due to the regularity properties of the solution, there exists a representative $z=[u, \varphi]$ such that $u \in C_{w}\left([0, \infty) ; G_{d i v}\right)$ and $\varphi \in C([0, \infty) ; H)$ (henceforth we shall always choose this representative). Therefore, (2.13) holds for all $t \geq 0$ since the function $\mathcal{E}(z(\cdot)):[0, \infty) \rightarrow \mathbb{R}$ is lower semicontinuous. The lower semicontinuity of $\mathcal{E}$ is a consequence of the fact that $F$ is a quadratic perturbation of a (strictly) convex function in $(-1,1)$. Indeeed, by (A6) we have that $F^{\prime \prime}(s) \geq \alpha_{*}$, for all $s \in(-1,1)$, with $\alpha_{*}=\alpha+\min _{[-1,1]} F_{2}^{\prime \prime}$. Then $F$ can be written in the form

$$
F(s)=G(s)+\frac{\alpha_{*}}{2} s^{2},
$$

with $G$ convex on $(-1,1)$ (see [21, Lemma 2]).

Let us now prove that the energy inequality (2.13) also holds between two arbitrary times $s$ and $t$. Indeed, setting

$$
\mathcal{E}_{\epsilon}\left(z_{\epsilon}(t)\right)=\frac{1}{2}\left\|u_{\epsilon}(t)\right\|^{2}+\frac{1}{2}\left\|\sqrt{a} \varphi_{\epsilon}(t)\right\|^{2}-\frac{1}{2}\left(\varphi_{\epsilon}(t), J * \varphi_{\epsilon}(t)\right)+\int_{\Omega} F_{\epsilon}\left(\varphi_{\epsilon}(t)\right)
$$

and applying [21, Lemma 3], we deduce (see Remark 6) that the approximate solution $z_{\epsilon}=\left[u_{\epsilon}, \varphi_{\epsilon}\right]$ satisfies

$$
\mathcal{E}_{\epsilon}\left(z_{\epsilon}(t)\right)+\int_{s}^{t}\left(2\left\|\sqrt{\nu\left(\varphi_{\epsilon}\right)} D u_{\epsilon}\right\|^{2}+\left\|\nabla \mu_{\epsilon}\right\|^{2}\right) d \tau \leq \mathcal{E}_{\epsilon}\left(z_{\epsilon}(s)\right)+\int_{s}^{t}\left\langle h, u_{\epsilon}\right\rangle d \tau
$$

for every $t \geq s$ and for a.e. $s \in(0, \infty)$, including $s=0$.

Define $G_{\epsilon}$ in such a way that

$$
F_{\epsilon}(s)=G_{\epsilon}(s)+\frac{\alpha_{*}}{2} s^{2},
$$

with $\alpha_{*}$ as in (3.54). Since, due to $(3.13), G_{\epsilon}$ is convex on $(-1,1)$, then we can write

$$
G_{\epsilon}\left(\varphi_{\epsilon}\right) \leq G_{\epsilon}(\varphi)+G_{\epsilon}^{\prime}\left(\varphi_{\epsilon}\right)\left(\varphi_{\epsilon}-\varphi\right)
$$


Hence, for every non-negative $\psi \in \mathcal{D}(0, t)$, we have

$$
\int_{Q_{t}} G_{\epsilon}\left(\varphi_{\epsilon}\right) \psi \leq \int_{Q_{t}} G_{\epsilon}(\varphi) \psi+\int_{Q_{t}} G_{\epsilon}^{\prime}\left(\varphi_{\epsilon}\right)\left(\varphi_{\epsilon}-\varphi\right) \psi
$$

where $Q_{t}:=\Omega \times(0, t)$. Thus, thanks to (3.39) and (3.41), we get

$$
\begin{aligned}
& \left|\int_{Q_{t}} G_{\epsilon}^{\prime}\left(\varphi_{\epsilon}\right)\left(\varphi_{\epsilon}-\varphi\right) \psi\right| \leq c\left\|G_{\epsilon}^{\prime}\left(\varphi_{\epsilon}\right)\right\|_{L^{2}(0, T ; H)}\left\|\varphi_{\epsilon}-\varphi\right\|_{L^{2}(0, T ; H)} \\
& \leq c\left\|\varphi_{\epsilon}-\varphi\right\|_{L^{2}(0, T ; H)} \rightarrow 0,
\end{aligned}
$$

as $\epsilon \rightarrow 0$. Here we have used the fact that, since $\left\|F_{\epsilon}^{\prime}\left(\varphi_{\epsilon}\right)\right\|_{L^{2}(0, T ; H)} \leq c$ and $G_{\epsilon}^{\prime}\left(\varphi_{\epsilon}\right)=$ $F_{\epsilon}^{\prime}\left(\varphi_{\epsilon}\right)-\alpha_{*} \varphi_{\epsilon}$, then $\left\|G_{\epsilon}^{\prime}\left(\varphi_{\epsilon}\right)\right\|_{L^{2}(0, T ; H)} \leq c$. Therefore, by using Lebesgue's theorem (recall (3.21) and the fact that $|\varphi|<1$ a.e. in $Q$ ) we find

$$
\limsup _{\epsilon \rightarrow 0} \int_{Q_{t}} G_{\epsilon}\left(\varphi_{\epsilon}\right) \psi \leq \lim _{\epsilon \rightarrow 0} \int_{Q_{t}} G_{\epsilon}(\varphi) \psi=\int_{Q_{t}} G(\varphi) \psi .
$$

On the other hand, thanks to Fatou's lemma and to the pointwise convergence $F_{\epsilon}\left(\varphi_{\epsilon}\right) \rightarrow F(\varphi)$, we also have the liminf inequality. Then, on account of (3.54) and (3.57), we deduce that

$$
\int_{Q_{t}} F_{\epsilon}\left(\varphi_{\epsilon}\right) \psi \rightarrow \int_{Q_{t}} F(\varphi) \psi, \quad \forall \psi \in \mathcal{D}(0, t), \psi \geq 0 .
$$

Let us multiply (3.56) by a non-negative $\psi \in \mathcal{D}(0, t)$ and integrate the resulting inequality w.r.t. $s$ from 0 and $t$, where $t>0$ is fixed. We obtain

$$
\begin{aligned}
& \mathcal{E}_{\epsilon}\left(z_{\epsilon}(t)\right) \int_{0}^{t} \psi(s) d s+\int_{0}^{t} \psi(s) \int_{s}^{t}\left(2\left\|\sqrt{\nu\left(\varphi_{\epsilon}\right)} D u_{\epsilon}\right\|^{2}+\left\|\nabla \mu_{\epsilon}\right\|^{2}\right) d \tau d s \\
& \leq \int_{0}^{t} \mathcal{E}_{\epsilon}\left(z_{\epsilon}(s)\right) \psi(s) d s+\int_{0}^{t} \psi(s) \int_{s}^{t}\left\langle h, u_{\epsilon}\right\rangle d \tau d s .
\end{aligned}
$$

By using strong and weak convergences for the sequence $\left\{z_{\epsilon}\right\}$ and (3.58), passing to the limit as $\epsilon \rightarrow 0$ in the above inequality, we infer

$$
\begin{aligned}
& \mathcal{E}(z(t)) \int_{0}^{t} \psi(s) d s+\int_{0}^{t} \psi(s) d s \int_{s}^{t}\left(2\|\sqrt{\nu(\varphi)} D u\|^{2}+\|\nabla \mu\|^{2}\right) d \tau \\
& \leq \int_{0}^{t} \mathcal{E}(z(s)) \psi(s) d s+\int_{0}^{t} \psi(s) d s \int_{s}^{t}\langle h, u\rangle d \tau
\end{aligned}
$$

which can be rewritten as follows

$$
V_{z}(t) \int_{0}^{t} \psi(s) d s \leq \int_{0}^{t} V_{z}(s) \psi(s) d s
$$

where

$$
V_{z}(t):=\mathcal{E}(z(t))+\int_{0}^{t}\left(2\|\sqrt{\nu(\varphi)} D u\|^{2}+\|\nabla \mu\|^{2}\right) d \tau-\int_{0}^{t}\langle h, u\rangle d \tau
$$

Thus we have

$$
\int_{0}^{t}\left(V_{z}(s)-V_{z}(t)\right) \psi(s) d s \geq 0, \quad \forall \psi \in \mathcal{D}(0, t), \psi \geq 0,
$$

which implies that $V_{z}(t) \leq V_{z}(s)$ for a.e. $s \in(0, t)$. Therefore, $(2.13)$ is proven. 
Finally, for $d=2$, we can choose

$\mu$ and $u$ as test functions in (2.10) and (2.11), respectively,

due to their regularity properties,

and then we can proceed as in [14, Proof of Corollary 2] to deduce (2.14). Indeed, when we consider the duality product $\left\langle\varphi_{t}, \mu\right\rangle$, we are led to the duality $\left\langle\varphi_{t}, G^{\prime}(\varphi)\right\rangle$, with the convex function $G$ given by (3.54). Now, define the functional $\mathcal{G}: H \rightarrow \mathbb{R} \cup\{+\infty\}$ as

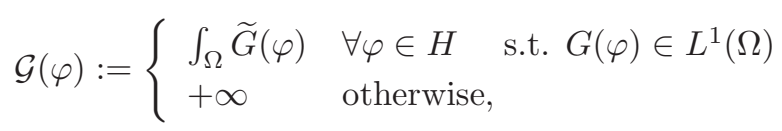

where $\widetilde{G}: \mathbb{R} \rightarrow \mathbb{R} \cup\{+\infty\}$ is given by $\widetilde{G}(s):=G(s)$ for all $s \in \operatorname{dom}(G)$, and $\widetilde{G}(s)=+\infty$ for all $s \in \mathbb{R}-\operatorname{dom}(G)$. The function $\widetilde{G}$ is a lower-semicontinuous proper convex function on $\mathbb{R}$ and, due to $(\mathrm{A} 7)$, its subdifferential is given by $\partial \widetilde{G}(s)=$ $G^{\prime}(s)$ for all $s \in \operatorname{int}(\operatorname{dom}(G))=(-1,1)$, and $\partial \widetilde{G}(s)=\emptyset$ for all $s \notin(-1,1)$. Then, [6, Proposition 2.8, Chap. II] entails that $\mathcal{G}$ is lower-semicontinuous and convex on $H$, and that $\xi \in \partial \mathcal{G}(\varphi)$ iff $\xi(x) \in \partial \widetilde{G}(\varphi(x))$ for a.a $x \in \Omega$. Notice that, if $|\varphi|<1$ a.e. in $\Omega$, the last condition is satisfied if and only if $\xi(x)=G^{\prime}(\varphi(x))$ for a.a. $x \in \Omega$. Let us now apply [16, Proposition 4.2] to the functional $\mathcal{G}$ and to the $\varphi$ component of the weak solution $z$ to (1.1)-(1.6). All conditions of this proposition are fulfilled, since, in particular, we have $G^{\prime}(\varphi) \in L^{2}(0, T ; V)$. Hence, we infer that $\mathcal{G}(\varphi(\cdot))$ is absolutely continuous on $[0, T]$ and that

$$
\frac{d}{d t} \mathcal{G}(\varphi(t))=\frac{d}{d t} \int_{\Omega} G(\varphi(t))=\left\langle\varphi_{t}(t), G^{\prime}(\varphi(t))\right\rangle, \quad \text { a.a. } t \in(0, T) .
$$

By exploiting this identity, the energy equation (2.14) can be obtained without difficulties.

REMARK 6. In [21, Lemma 3] a growth assumption is made on the regular potential (polynomial growth less then 6 when $d=3$ ). Therefore, the application of [21, Lemma 3] to obtain the approximate energy inequality (3.56) would require the condition $q=1$ (recall that the approximate potential $F_{\epsilon}$ has polynomial growth of order $2+2 q$ ). Nevertheless, by exploiting an argument of the same kind as above and by suitably approximating regular potentials of arbitrary polynomial growth by a sequence of potentials of polynomial growth of order less then 6 , it is not difficult to improve [21, Lemma 3] and remove such growth assumption. Therefore [21, Lemma 3] can be extended to regular potentials of arbitrary polynomial growth and (3.56) also holds for $q>1$.

\section{Global attractor in $2 \mathrm{D}$}

In this section we first prove that in $2 \mathrm{D}$ we can define a generalized semiflow on a suitable metric space $\mathcal{X}_{m_{0}}$ which is point dissipative and eventually bounded. Furthermore, we show that such generalized semiflow possesses a (unique) global 
attractor, provided that the potential $F$ is bounded in $(-1,1)$ (like, e.g., (1.7)). The argument is a generalization of the one used in $[\mathbf{2 1}]$ and based on [5]. Henceforth, we refer to [5] for the basic definitions and results on the theory of generalized semiflows.

Consider system (1.1)-(1.4) endowed with (1.5) for $d=2$ and assume that the external force $h$ is time-independent, i.e.,

(A9): $h \in V_{d i v}^{\prime}$.

The first step is to define a suitable metric space for the weak solutions and consequently to construct a generalized semiflow. To this aim, fix $m_{0} \in(0,1)$ and introduce the metric space

$$
\mathcal{X}_{m_{0}}:=G_{d i v} \times \mathcal{Y}_{m_{0}},
$$

where

$$
\mathcal{Y}_{m_{0}}:=\left\{\varphi \in L^{\infty}(\Omega):|\varphi|<1 \text { a.e. in } \Omega, \quad F(\varphi) \in L^{1}(\Omega), \quad|\bar{\varphi}| \leq m_{0}\right\} .
$$

The space $\mathcal{X}_{m_{0}}$ is endowed with the metric

$$
\boldsymbol{d}\left(z_{1}, z_{2}\right):=\left\|u_{1}-u_{2}\right\|+\left\|\varphi_{1}-\varphi_{2}\right\|+\left|\int_{\Omega} F\left(\varphi_{1}\right)-\int_{\Omega} F\left(\varphi_{2}\right)\right|^{1 / 2},
$$

for every $z_{1}:=\left[u_{1}, \varphi_{1}\right]$ and $z_{2}:=\left[u_{2}, \varphi_{2}\right]$ in $\mathcal{X}_{m_{0}}$. Let us denote by $\mathcal{G}$ the set of all weak solutions corresponding to all initial data $z_{0}=\left[u_{0}, \varphi_{0}\right] \in \mathcal{X}_{m_{0}}$. We prove that $\mathcal{G}$ is a generalized semiflow on $\mathcal{X}_{m_{0}}$.

Proposition 2. Let $d=2$ and suppose that (A1)-(A7) and (A9) hold. Then $\mathcal{G}$ is a generalized semiflow on $\mathcal{X}_{m_{0}}$.

Proof. It can be seen immediately that hypotheses (H1), (H2) and (H3) of the definition of generalized semiflow [5, Definition 2.1] are satisfied. It remains to prove the upper semicontinuity with respect to initial data, i.e., that $\mathcal{G}$ satisfies (H4) of [5, Definition 2.1]. We can argue as in [21, Proposition 3]. Thus we only give the main steps of the proof. Consider a sequence $\left\{z_{j}\right\} \subset \mathcal{G}$, with $z_{j}:=\left[u_{j}, \varphi_{j}\right]$ such that $z_{j}(0):=\left[u_{j 0}, \varphi_{j 0}\right] \rightarrow z_{0}:=\left[u_{0}, \varphi_{0}\right]$ in $\mathcal{X}_{m_{0}}$. We have to show that there exist a subsequence $\left\{z_{j_{k}}\right\}$ and a weak solution $z \in \mathcal{G}$ with $z(0)=z_{0}$ such that $z_{j_{k}}(t) \rightarrow z(t)$ for each $t \geq 0$. Now, every weak solution $z_{j}$ satisfies the energy identity (2.14) so that

$$
\mathcal{E}\left(z_{j}(t)\right)+\int_{0}^{t}\left(2\left\|\sqrt{\nu\left(\varphi_{j}\right)} D u_{j}(\tau)\right\|^{2}+\left\|\nabla \mu_{j}(\tau)\right\|^{2}\right) d \tau=\mathcal{E}\left(z_{j 0}\right)+\int_{0}^{t}\left\langle h, u_{j}(\tau)\right\rangle d \tau,
$$

where $z_{j 0}:=z_{j}(0)$. From this identity and using the assumptions on $F$ we deduce estimates of the form (3.22)-(3.28). Furthermore, since $\left|\varphi_{0 j}\right| \leq m_{0}$ and $m_{0} \in(0,1)$ is fixed, we can repeat the argument used in the existence proof to control the 
sequence of the averages of the approximated chemical potentials (see (3.29)-(3.38)) and get

$$
\left\|F^{\prime}\left(\varphi_{j}\right)\right\|_{L^{2}\left(0, T ; L^{1}(\Omega)\right)} \leq L\left(m_{0}\right),
$$

where $L\left(m_{0}\right)$ is a positive constant depending on $m_{0}$. Hence, an estimate of the form (3.39) for $\mu_{j}$ holds. From these estimates we deduce the existence of a couple $z=[u, \varphi]$ and of a function $\mu$ with $u, \varphi$ and $\mu$ having the regularity properties (2.3)-(2.8) and such that (3.40)-(3.47) hold for suitable subsequences of $\left\{u_{j}\right\},\left\{\varphi_{j}\right\}$ and $\left\{\mu_{j}\right\}$. In order to prove that $z=[u, \varphi]$ is a weak solution by passing to the limit in the variational formulation for $z_{j}$ we need to know that (2.9) is satisfied for $\varphi$. To this aim we use the same argument we applied to the sequence of approximate solutions $\left\{\varphi_{\epsilon}\right\}$ (cf. proof of Theorem 1).

More precisely, for $\eta \in(0,1)$ fixed

we can introduce the sets

$E_{1, \eta}^{j}:=\left\{(x, t) \in Q: \varphi_{j}(x, t)>1-\eta\right\}, \quad E_{2, \eta}^{j}:=\left\{(x, t) \in Q: \varphi_{j}(x, t)<-1+\eta\right\}$, and so we have

$$
H^{\prime}(1-\eta)\left|E_{1, \eta}^{j}\right|_{d+1} \leq\left\|H^{\prime}\left(\varphi_{j}\right)\right\|_{L^{1}(Q)}, \quad\left|H^{\prime}(-1+\eta)\right|\left|E_{2, \eta}^{j}\right|_{d+1} \leq\left\|H^{\prime}\left(\varphi_{j}\right)\right\|_{L^{1}(Q)},
$$

where $H$ is defined as in (3.35). Therefore, recalling (4.5), by first letting $j \rightarrow \infty$ and then $\eta \rightarrow 0$ we can deduce that

$$
|\varphi(x, t)|<1 \quad \text { for a.e. }(x, t) \in Q .
$$

On the other hand, since we also have

$$
u_{j}(t) \rightarrow u(t) \quad \text { weakly in } G_{d i v}, \quad \varphi_{j}(t) \rightarrow \varphi(t) \quad \text { weakly in } H, \quad \forall t \geq 0,
$$

then $z(0)=z_{0}$. It remains to prove the convergence of the sequence $\left\{z_{j}(t)\right\}$ to $z(t)$ in $\mathcal{X}_{m_{0}}$ for each $t \geq 0$. Reasoning as in [21], we represent the singular potential $\mathrm{F}$ as follows

$$
F(s)=G(x, s)-\left(a(x)-\frac{c_{0}}{2}\right) \frac{s^{2}}{2},
$$

where $c_{0}=\alpha+\beta+\min _{[-1,1]} F_{2}^{\prime \prime}>0$. Here, due to $(\mathrm{A} 6)$, the function $G(x, \cdot)$ is strictly convex in $(-1,1)$ for a.e. $x \in \Omega$. Therefore, the energy $\mathcal{E}$ can still be written as

$$
\mathcal{E}(z)=\frac{1}{2}\|u\|^{2}+\frac{c_{0}}{4}\|\varphi\|^{2}-\frac{1}{2}(\varphi, J * \varphi)+\int_{\Omega} G(x, \varphi(x)) d x, \quad \forall z=[u, \varphi] \in \mathcal{X}_{m_{0}},
$$

and the same argument used in [21, Proposition 3] applies.

As done for regular potentials (see [21]), a dissipativity property of the generalized semiflow $\mathcal{G}$ can be proven in the case of singular (bounded) potentials.

Proposition 3. Let $d=2$ and suppose that (A1)-(A\%), (A9) hold. Then $\mathcal{G}$ is point dissipative and eventually bounded. 
PROOF. Recalling the proof of [14, Corollary 2] a dissipative estimate can be established, namely,

$$
\mathcal{E}(z(t)) \leq \mathcal{E}\left(z_{0}\right) e^{-k t}+F\left(\overline{\varphi_{0}}\right)|\Omega|+K, \quad \forall t \geq 0,
$$

where $k, K$ are two positive constants which are independent of the initial data, with $K$ depending on $\Omega, \nu_{1}, J, F,\|h\|_{V_{d i v}^{\prime}}$. From (4.6) we get (see [21, Proposition 4])

$$
\boldsymbol{d}^{2}(z(t), 0) \leq c \mathcal{E}\left(z_{0}\right) e^{-k t}+c M_{m_{0}}+c, \quad \forall t \geq 0,
$$

which entails that the generalized semiflow $\mathcal{G}$ is point dissipative and eventually bounded.

We can now state the main result of this section.

Proposition 4. Let $d=2$ and suppose that (A1)-(A7), (A9) hold. Furthermore, assume that $F$ is bounded in $(-1,1)$. Then $\mathcal{G}$ possesses a global attractor.

Proof. In light of Proposition 3 and by [5, Proposition 3.2] and [5, Theorem 3.3] we only need to show that $\mathcal{G}$ is compact. Let $\left\{z_{j}\right\} \subset \mathcal{G}$ be a sequence with $\left\{z_{j}(0)\right\}$ bounded in $\mathcal{X}_{m_{0}}$. We claim that there exists a subsequence $\left\{z_{j_{k}}\right\}$ such that $z_{j_{k}}(t)$ converges in $\mathcal{X}_{m_{0}}$ for every $t>0$. Indeed, the energy identity (4.4) entails the existence of a subsequence (not relabeled) such that (see the proof of Proposition 2) for almost all $t>0$

$$
u_{j}(t) \rightarrow u(t) \quad \text { strongly in } G_{d i v}, \quad \varphi_{j}(t) \rightarrow \varphi(t) \quad \text { strongly in } H \text { and a.e. in } \Omega,
$$

where $z=[u, \varphi]$ is a weak solution. Since $F$ is bounded in $(-1,1)$, by Lebesgue's theorem we therefore have

$$
\int_{\Omega} F\left(\varphi_{j}(t)\right) \rightarrow \int_{\Omega} F(\varphi(t)), \quad \text { a.e. } t>0 .
$$

Hence $\mathcal{E}\left(z_{j}(t)\right) \rightarrow \mathcal{E}(z(t))$ for almost all $t>0$. Thus, arguing as in $[\mathbf{2 1}$, Theorem 3 , Proposition 3], we deduce that $z_{j}(t) \rightarrow z(t)$ in $\mathcal{X}_{m_{0}}$ for all $t>0$, which yields the compactness of $\mathcal{G}$.

We can also prove the existence of the global attractor for the convective nonlocal Cahn-Hilliard equation with $u \in L^{\infty}(\Omega)^{d} \cap V_{d i v}, d=2,3$. Indeed, thanks to Proposition 1, we can define a semigroup $S(t)$ on $\mathcal{Y}_{m_{0}}$ (cf. (4.2)) endowed the metric

$$
\overline{\mathbf{d}}\left(\varphi_{1}, \varphi_{2}\right)=\left\|\varphi_{1}-\varphi_{2}\right\|+\left|\int_{\Omega} F\left(\varphi_{1}\right)-\int_{\Omega} F\left(\varphi_{2}\right)\right|^{1 / 2}, \quad \forall \varphi_{1}, \varphi_{2} \in \mathcal{Y}_{m_{0}} .
$$

Then we have

Theorem 2. Let $u \in L^{\infty}(\Omega)^{d} \cap V_{\text {div }}$ be given. Suppose that (A1), (A3)-(A7) are satisfied and assume that $F$ is bounded in $(-1,1)$. Then the dynamical system $\left(\mathcal{Y}_{m_{0}}, S(t)\right)$ possesses a connected global attractor. 
The proof goes as in [21, Proof of Theorem 4]. Note that, due to uniqueness, the global attractor is connected.

\section{Existence of a trajectory attractor}

In this section, by relying on the theory developed in $[\mathbf{1 1}, \mathbf{1 2}]$ (see also [34]), we prove that a trajectory attractor can be constructed for the nonlocal Cahn-HilliardNavier-Stokes system (1.1)-(1.4) subject to (1.5) with $F$ satisfying (A3)-(A7). The construction of the trajectory attractor for problem (1.1)-(1.5) in the case of regular potentials with polynomial growth has been done in $[\mathbf{2 1}]$. We concentrate on the 3D case.

Let us first resume some basic definitions and results from the theory of trajectory attractors for non-autonomous evolution equations (see [12, Chap. XI and Chap. XIV] and [11] for details).

Consider an abstract nonlinear non-autonomous evolution equation with symbol $\sigma$ in a set $\Sigma$. The symbol $\sigma$ is a functional parameter which represents all time-dependent terms (like external forces) and coefficients of the equation.

For every $M>0$, the solutions are sought in a topological (usually Banach) space $\mathcal{F}_{M}$ which consists of vector-valued functions $z:[0, M] \rightarrow E$, where $E$ is a given Banach space. The space $\mathcal{F}_{M}$ is endowed with a given topology $\Theta_{M}$, such that $\left(\mathcal{F}_{M}, \Theta_{M}\right)$ is a Hausdorff topological space with a countable base. By means of $\mathcal{F}_{M}$ the space $\mathcal{F}_{\text {loc }}^{+}$is defined as $\mathcal{F}_{\text {loc }}^{+}:=\left\{z:[0, \infty) \rightarrow E: \Pi_{[0, M]} z \in \mathcal{F}_{M}\right.$, for all $\left.M>0\right\}$, where $\Pi_{[0, M]}$ is the restriction operator on the interval $[0, M]$. The space $\mathcal{F}_{l o c}^{+}$is endowed with a local convergence topology $\Theta_{l o c}^{+}$, i.e., the topology that induces the following definition of convergence for a sequence $\left\{z_{n}\right\} \subset \mathcal{F}_{\text {loc }}^{+}$to $z \in \mathcal{F}_{\text {loc }}^{+}$

$$
z_{n} \rightarrow z \quad \text { in } \Theta_{l o c}^{+} \quad \text { if } \quad \Pi_{[0, M]} z_{n} \rightarrow \Pi_{[0, M]} z \quad \text { in } \Theta_{M}
$$

for every $M>0$. It can be seen that the space $\left(\mathcal{F}_{l o c}^{+}, \Theta_{l o c}\right)$ is a Hausdorff topological space with a countable base. On the space $\mathcal{F}_{\text {loc }}^{+}$the translation semigroup $\{T(t)\}_{t \geq 0}$ is defined, for every $z \in \mathcal{F}_{l o c}^{+}$, as

$$
T(t) z:=z(\cdot+t), \quad \forall t \geq 0 .
$$

The semigroup $\{T(t)\}$ is continuous in the topology $\Theta_{l o c}^{+}$(see, e.g., $[\mathbf{1 1}$, Proposition $2.2])$.

For each $\sigma \in \Sigma$ let us denote by $\mathcal{K}_{\sigma}^{M}$ the set of some solutions from $\mathcal{F}_{M}$ and by $\mathcal{K}_{\sigma}^{+}$the set of some solutions from $\mathcal{F}_{\text {loc }}^{+}$. The set $\mathcal{K}_{\sigma}^{+}$is said to be a trajectory space of the evolution equation corresponding to the symbol $\sigma \in \Sigma$.

We shall need a slightly more general functional setting than the one devised in $[\mathbf{1 1}]$. Indeed, in order to construct a trajectory attractor without any boundedness assumption on the potential $F$, we must define a family of bounded sets of trajectories with a suitable attraction property. The idea is to take a subspace $\mathcal{F}_{b}^{+}$ of the space $\mathcal{F}_{\text {loc }}^{+}$on which a metric $d_{\mathcal{F}_{b}^{+}}$is given and assume that the trajectory 
space $\mathcal{K}_{\sigma}^{+}$corresponding to the symbol $\sigma \in \Sigma$ satisfies $\mathcal{K}_{\sigma}^{+} \subset \mathcal{F}_{b}^{+}$, for every $\sigma \in \Sigma$. This approach is in the spirit of the theory of $(\mathcal{M}, \mathcal{T})$-attractors in $[\mathbf{1 2}$, Chap. XI, Section 3], where $\mathcal{T}$ is a topological space where some metric is defined and $\mathcal{M}$ is the corresponding metric space.

Consider the united trajectory space $\mathcal{K}_{\Sigma}^{+}:=\cup_{\sigma \in \Sigma} \mathcal{K}_{\sigma}^{+}$of the family $\left\{\mathcal{K}_{\sigma}^{+}\right\}_{\sigma \in \Sigma}$. We have $\mathcal{K}_{\Sigma}^{+} \subset \mathcal{F}_{b}^{+}$. Recall that the family $\left\{\mathcal{K}_{\sigma}^{+}\right\}_{\sigma \in \Sigma}$ is said to be translationcoordinated if for any $\sigma \in \Sigma$ and any $z \in \mathcal{K}_{\sigma}^{+}$we have $T(t) z \in \mathcal{K}_{T(t) \sigma}^{+}$, for all $t \geq 0$. If $\left\{\mathcal{K}_{\sigma}^{+}\right\}_{\sigma \in \Sigma}$ is translation-coordinated, then we have $T(t) \mathcal{K}_{\Sigma}^{+} \subset \mathcal{K}_{\Sigma}^{+}$, for every $t \geq 0$, i.e., the translation semigroup $\{T(t)\}$ acts on $\mathcal{K}_{\Sigma}^{+}$. Introduce now the family

$$
\mathcal{B}_{\Sigma}^{+}:=\left\{B \subset \mathcal{K}_{\Sigma}^{+}: B \text { bounded in } \mathcal{F}_{b}^{+} \text {w.r.t. the metric } d_{\mathcal{F}_{b}^{+}}\right\} .
$$

We shall refer to this family in the definition of a uniformly (w.r.t $\sigma \in \Sigma$ ) attracting set $P \subset \mathcal{F}_{\text {loc }}^{+}$for $\left\{\mathcal{K}_{\sigma}^{+}\right\}_{\sigma \in \Sigma}$ in the topology $\Theta_{\text {loc }}^{+}$and in the definition of the uniform (w.r.t. $\sigma \in \Sigma$ ) trajectory attractor $\mathcal{A}_{\Sigma}$ of the translation semigroup $\{T(t)\}$.

Definition 2. A set $P \subset \mathcal{F}_{\text {loc }}^{+}$is said to be a uniformly (w.r.t. $\sigma \in \Sigma$ ) attracting set for the family $\left\{\mathcal{K}_{\sigma}^{+}\right\}_{\sigma \in \Sigma}$ in the topology $\Theta_{\text {loc }}^{+}$if $P$ is uniformly (w.r.t. $\sigma \in \Sigma)$ attracting for the family $\mathcal{B}_{\Sigma}^{+}$, i.e. for any $B \in \mathcal{B}_{\Sigma}^{+}$and for any neighbourhood $\mathcal{O}(P)$ in $\Theta_{\text {loc }}^{+}$there exists $t_{1} \geq 0$ such that $T(t) B \subset \mathcal{O}(P)$, for every $t \geq t_{1}$.

Definition 3. A set $\mathcal{A}_{\Sigma} \subset \mathcal{F}_{\text {loc }}^{+}$is said to be a uniform (w.r.t. $\sigma \in \Sigma$ ) trajectory attractor of the translation semigroup $\{T(t)\}$ in the topology $\Theta_{\text {loc }}^{+}$if $\mathcal{A}_{\Sigma}$ is compact in $\Theta_{\text {loc }}^{+}, \mathcal{A}_{\Sigma}$ is a uniformly (w.r.t. $\sigma \in \Sigma$ ) attracting set for $\left\{\mathcal{K}_{\sigma}^{+}\right\}_{\sigma \in \Sigma}$ in the topology $\Theta_{\text {loc }}^{+}$, and $\mathcal{A}_{\Sigma}$ is the minimal compact and uniformly (w.r.t. $\sigma \in \Sigma$ ) attracting set for the family $\left\{\mathcal{K}_{\sigma}^{+}\right\}_{\sigma \in \Sigma}$ in the topology $\Theta_{\text {loc }}^{+}$, i.e., if $P$ is any compact uniformly (w.r.t. $\sigma \in \Sigma$ ) attracting set for the family $\left\{\mathcal{K}_{\sigma}^{+}\right\}_{\sigma \in \Sigma}$, then $\mathcal{A}_{\Sigma} \subset P$.

From the definition it follows that, if the trajectory attractor exists, then it is unique.

To prove some properties of the trajectory attractor we need that the set $\mathcal{K}_{\Sigma}^{+}$ be closed in $\Theta_{l o c}^{+}$. Assume that $\Sigma$ is a complete metric space. Recall that the family $\left\{\mathcal{K}_{\sigma}^{+}\right\}_{\sigma \in \Sigma}$ is called $\left(\Theta_{\text {loc }}^{+}, \Sigma\right)$-closed if the graph set $\cup_{\sigma \in \Sigma} \mathcal{K}_{\sigma}^{+} \times\{\sigma\}$ is closed in the topological space $\Theta_{\text {loc }}^{+} \times \Sigma$. If $\left\{\mathcal{K}_{\sigma}^{+}\right\}_{\sigma \in \Sigma}$ is $\left(\Theta_{\text {loc }}^{+}, \Sigma\right)$-closed and $\Sigma$ is compact, then $\mathcal{K}_{\Sigma}^{+}$is closed in $\Theta_{\text {loc }}^{+}$(see, e.g., [11, Proposition 3.2]).

Remark 7. We shall see that (cf. Proposition 6), although by means of the topological-metric scheme above the boundedness assumption on the potential $F$ can be avoided as far as the construction of the trajectory attractor for system (1.1)-(1.5) with singular potential is concerned, it seems difficult to get rid of such an assumption when one wants to prove the closedness of the trajectory space $\mathcal{K}_{\Sigma}^{+}$.

We now state the main abstract result which can be established by applying $\left[\mathbf{1 2}\right.$, Chap. XI, Theorem 2.1] to the topological space $\mathcal{F}_{l o c}^{+}$, to the family $\mathcal{B}_{\Sigma}^{+}$and 
to the family

$$
\mathcal{B}_{\omega(\Sigma)}^{+}:=\left\{B \subset \mathcal{K}_{\omega(\Sigma)}^{+}: B \text { bounded in } \mathcal{F}_{b}^{+} \text {w.r.t. the metric } d_{\mathcal{F}_{b}^{+}}\right\}
$$

where $\mathcal{K}_{\omega(\Sigma)}^{+}:=\cup_{\sigma \in \omega(\Sigma)} \mathcal{K}_{\sigma}^{+}$and where $\omega(\Sigma)$ is the $\omega$-limit set of $\Sigma$, defined as

$$
\omega(\Sigma):=\bigcap_{t \geq 0}\left[\bigcup_{h \geq t} T(h) \Sigma\right]_{\Sigma}=\left\{\sigma \in \Sigma: \exists h_{n} \rightarrow \infty, \exists \sigma_{n} \in \Sigma \text { s.t. } T\left(h_{n}\right) \sigma_{n} \rightarrow \sigma\right\} .
$$

We also refer the reader to $[\mathbf{1 1}$, Theorem 3.1].

THEOREM 3. Let the spaces $\left(\mathcal{F}_{\text {loc }}^{+}, \Theta_{\text {loc }}^{+}\right)$and $\left(\mathcal{F}_{b}^{+}, d_{\mathcal{F}_{b}^{+}}\right)$be as above, and the family of trajectory spaces $\left\{\mathcal{K}_{\sigma}^{+}\right\}_{\sigma \in \Sigma}$ corresponding to the evolution equation with symbols $\sigma \in \Sigma$ be such that $\mathcal{K}_{\sigma}^{+} \subset \mathcal{F}_{b}^{+}$, for every $\sigma \in \Sigma$. Assume there exists a subset $P \subset \mathcal{F}_{\text {loc }}^{+}$which is compact in $\Theta_{\text {loc }}^{+}$and uniformly (w.r.t. $\sigma \in \Sigma$ ) attracting in $\Theta_{\text {loc }}^{+}$ for the family $\left\{\mathcal{K}_{\sigma}^{+}\right\}_{\sigma \in \Sigma}$ in the topology $\Theta_{\text {loc }}^{+}$. Then, the translation semigroup $\{T(t)\}_{t \geq 0}$, which acts on $\mathcal{K}_{\Sigma}^{+}$if the family $\left\{\mathcal{K}_{\sigma}^{+}\right\}_{\sigma \in \Sigma}$ is translation-coordinated, possesses a (unique) uniform (w.r.t. $\sigma \in \Sigma$ ) trajectory attractor $\mathcal{A}_{\Sigma} \subset P$ which is strictly invariant

$$
T(t) \mathcal{A}_{\Sigma}=\mathcal{A}_{\Sigma}, \quad \forall t \geq 0 .
$$

In addition, if the family $\left\{\mathcal{K}_{\sigma}^{+}\right\}_{\sigma \in \Sigma}$ is translation-coordinated and $\left(\Theta_{\text {loc }}^{+}, \Sigma\right)-$ closed, with $\Sigma$ a compact metric space, then $\mathcal{A}_{\Sigma} \subset \mathcal{K}_{\Sigma}^{+}$and

$$
\mathcal{A}_{\Sigma}=\mathcal{A}_{\omega(\Sigma)},
$$

where $\mathcal{A}_{\omega(\Sigma)}$ is the uniform (w.r.t. $\sigma \in \omega(\Sigma)$ ) trajectory attractor for the family $\mathcal{B}_{\omega(\Sigma)}^{+}$and $\mathcal{A}_{\omega(\Sigma)} \subset \mathcal{K}_{\omega(\Sigma)}^{+}$.

Suppose that for a given abstract nonlinear non-autonomous evolution equation a dissipative estimate of the following form can be established

$$
d_{\mathcal{F}_{b}^{+}}\left(T(t) w, w_{0}\right) \leq \Lambda_{0}\left(d_{\mathcal{F}_{b}^{+}}\left(w, w_{0}\right)\right) e^{-k t}+\Lambda_{1}, \quad \forall t \geq t_{0},
$$

for every $w \in \mathcal{K}_{\Sigma}^{+}$, for some fixed $w_{0} \in \mathcal{F}_{b}^{+}$and for some $\Lambda_{0}:[0, \infty) \rightarrow[0, \infty)$ locally bounded and some constants $\Lambda_{1} \geq 0, k>0$, where $k, \Lambda_{0}$ and $\Lambda_{1}$ are independent of $w$. Furthermore, assume that the ball

$$
B_{\mathcal{F}_{b}^{+}}\left(w_{0}, 2 \Lambda_{1}\right):=\left\{w \in \mathcal{F}_{b}^{+}: d_{\mathcal{F}_{b}^{+}}\left(w, w_{0}\right) \leq 2 \Lambda_{1}\right\}
$$

is compact in $\Theta_{l o c}^{+}$. By virtue of (5.1) such ball is a uniformly (w.r.t. $\sigma \in \Sigma$ ) attracting set for the family $\left\{\mathcal{K}_{\sigma}^{+}\right\}_{\sigma \in \Sigma}$ in the topology $\Theta_{\text {loc }}^{+}$(actually, $B_{\mathcal{F}_{b}^{+}}\left(w_{0}, 2 \Lambda_{1}\right)$ is uniformly (w.r.t. $\sigma \in \Sigma$ ) absorbing for the family $\mathcal{B}_{\Sigma}^{+}$). Theorem 3 therefore entails that the translation semigroup $\{T(t)\}_{t \geq 0}$ possesses a (unique) uniform (w.r.t. $\sigma \in \Sigma)$ trajectory attractor $\mathcal{A}_{\Sigma} \subset B_{\mathcal{F}_{b}^{+}}\left(w_{0}, 2 \Lambda_{1}\right)$.

Let us now turn to (1.1)-(1.5) and apply to this system the scheme described above. 
For $q \geq 1, m_{0} \in(0,1)$ and for any given $M>0$ we set

$$
\begin{gathered}
\mathcal{F}_{M}=\left\{[v, \psi] \in L^{\infty}\left(0, M ; G_{d i v} \times L^{2+2 q}(\Omega)\right) \cap L^{2}\left(0, M ; V_{d i v} \times V\right):\right. \\
v_{t} \in L^{4 / 3}\left(0, M ; V_{d i v}^{\prime}\right), \psi_{t} \in L^{2}\left(0, M ; V^{\prime}\right), \\
\left.\psi \in L^{\infty}\left(Q_{M}\right),|\psi| \leq 1 \text { a.e. in } Q_{M},|\bar{\psi}| \leq m_{0}\right\},
\end{gathered}
$$

where $Q_{M}=\Omega \times(0, M)$. We endow $\mathcal{F}_{M}$ with the weak topology $\Theta_{M}$ which induces the following notion of weak convergence: a sequence $\left\{\left[v_{m}, \psi_{m}\right]\right\} \subset \mathcal{F}_{M}$ is said to converge to $[v, \psi] \in \mathcal{F}_{M}$ in $\Theta_{M}$ if

$$
\begin{aligned}
& v_{n} \rightarrow v \quad \text { weakly }^{*} \text { in } L^{\infty}\left(0, M ; G_{d i v}\right) \text { and weakly in } L^{2}\left(0, M ; V_{d i v}\right), \\
& \left(v_{n}\right)_{t} \rightarrow v_{t} \quad \text { weakly in } L^{4 / 3}\left(0, M ; V_{d i v}^{\prime}\right), \\
& \psi_{n} \rightarrow \psi \quad \text { weakly* in } L^{\infty}\left(0, M ; L^{2+2 q}(\Omega)\right) \text { and weakly in } L^{2}(0, M ; V), \\
& \left(\psi_{n}\right)_{t} \rightarrow \psi_{t} \quad \text { weakly in } L^{2}\left(0, M ; V^{\prime}\right) .
\end{aligned}
$$

Then, we can define the space

$$
\begin{gathered}
\mathcal{F}_{l o c}^{+}=\left\{[v, \psi] \in L_{l o c}^{\infty}\left([0, \infty) ; G_{d i v} \times L^{2+2 q}(\Omega)\right) \cap L_{l o c}^{2}\left([0, \infty) ; V_{d i v} \times V\right):\right. \\
v_{t} \in L_{l o c}^{4 / 3}\left([0, \infty) ; V_{d i v}^{\prime}\right), \psi_{t} \in L_{l o c}^{2}\left([0, \infty) ; V^{\prime}\right), \\
\left.\psi \in L^{\infty}\left(Q_{M}\right),|\psi| \leq 1 \text { a.e. in } Q_{M}, \forall M>0,|\bar{\psi}| \leq m_{0}\right\},
\end{gathered}
$$

endowed with the inductive limit weak topology $\Theta_{l o c}^{+}$. In $\mathcal{F}_{\text {loc }}^{+}$we consider the following subset

$$
\begin{aligned}
\mathcal{F}_{b}^{+}=\{ & {[v, \psi] \in L^{\infty}\left(0, \infty ; G_{d i v} \times L^{2+2 q}(\Omega)\right) \cap L_{t b}^{2}\left(0, \infty ; V_{d i v} \times V\right): } \\
& v_{t} \in L_{t b}^{4 / 3}\left(0, \infty ; V_{d i v}^{\prime}\right), \psi_{t} \in L_{t b}^{2}\left(0, \infty ; V^{\prime}\right), \\
& \left.\psi \in L^{\infty}\left(Q_{\infty}\right),|\psi|<1 \text { a.e. in } Q_{\infty},|\bar{\psi}| \leq m_{0}, F(\psi) \in L^{\infty}\left(0, \infty ; L^{1}(\Omega)\right)\right\},
\end{aligned}
$$

where $Q_{\infty}:=\Omega \times(0, \infty)$, endowed with the following metric

$$
\begin{aligned}
d_{\mathcal{F}_{b}^{+}}\left(z_{2}, z_{1}\right): & =\left\|z_{2}-z_{1}\right\|_{L^{\infty}\left(0, \infty ; G_{d i v} \times L^{2+2 q}(\Omega)\right)}+\left\|z_{2}-z_{1}\right\|_{L_{t b}^{2}\left(0, \infty ; V_{d i v} \times V\right)} \\
& +\left\|\left(v_{2}\right)_{t}-\left(v_{1}\right)_{t}\right\|_{L_{t b}^{4 / 3}\left(0, \infty ; V_{d i v}^{\prime}\right)}+\left\|\left(\psi_{2}\right)_{t}-\left(\psi_{1}\right)_{t}\right\|_{L_{t b}^{2}\left(0, \infty ; V^{\prime}\right)} \\
& +\left\|\int_{\Omega} F\left(\psi_{2}\right)-\int_{\Omega} F\left(\psi_{1}\right)\right\|_{L^{\infty}(0, \infty)}^{1 / 2}
\end{aligned}
$$

for all $z_{2}:=\left[v_{2}, \psi_{2}\right], z_{1}:=\left[v_{1}, \psi_{1}\right] \in \mathcal{F}_{b}^{+}$. If $X$ is a Banach space and $\tau \in \mathbb{R}$, we denote by $L_{t b}^{p}(\tau, \infty ; X), 1 \leq p<\infty$, the space of functions $f \in L_{l o c}^{p}([\tau, \infty) ; X)$ that are translation bounded in $L_{l o c}^{p}([\tau, \infty) ; X)$, i.e. such that (see, e.g., [12])

$$
\|f\|_{L_{t b}^{p}(\tau, \infty ; X)}^{p}:=\sup _{t \geq \tau} \int_{t}^{t+1}\|f(s)\|_{X}^{p} d s<\infty .
$$

For the trajectory space $\mathcal{K}_{h}^{+}$corresponding to a symbol $h$ we mean 
Definition 4. For every $h \in L_{\text {loc }}^{2}\left([0, \infty) ; V_{\text {div }}^{\prime}\right)$ the trajectory space $\mathcal{K}_{h}^{+}$is the set of all weak solutions $z=[v, \psi]$ to (1.1)-(1.5) with external force $h$ which belong to the space $\mathcal{F}_{\text {loc }}^{+}$and satisfy the energy inequality (2.13) for all $t \geq s$ and for a.a. $s \in(0, \infty)$.

REMARK 8. Notice that in the definition of the trajectory space $\mathcal{K}_{h}^{+}$we do not assume that the energy inequality $(2.13)$ is satisfied also for $s=0$. In this way the family $\left\{\mathcal{K}_{h}^{+}\right\}_{h \in \Sigma}\left(\Sigma\right.$ is a generic symbol space included in $\left.L_{\text {loc }}^{2}\left([0, \infty) ; V_{\text {div }}^{\prime}\right)\right)$ is translation-coordinated and therefore the semigroup $\{T(t)\}$ acts on $\mathcal{K}_{\Sigma}^{+}$.

According to Theorem 1, if (A1)-(A7) hold, then for every $z_{0}=\left[v_{0}, \psi_{0}\right]$ such that

$v_{0} \in G_{d i v}, \quad \psi_{0} \in L^{\infty}(\Omega), \quad\left\|\psi_{0}\right\|_{\infty} \leq 1, \quad F\left(\psi_{0}\right) \in L^{1}(\Omega), \quad\left|\bar{\psi}_{0}\right| \leq m_{0}$, and every $h$ satisfying (A8) there exists a trajectory $z \in \mathcal{K}_{h}^{+}$for which $z(0)=z_{0}$.

Let us consider now

$$
h_{0} \in L_{t b}^{2}\left(0, \infty ; V_{d i v}^{\prime}\right),
$$

and observe that $h_{0}$ is translation compact in $L_{l o c, w}^{2}\left([0, \infty) ; V_{d i v}^{\prime}\right)$. Then, by definition, the hull

$$
\mathcal{H}_{+}\left(h_{0}\right):=\left[\left\{T(t) h_{0}: t \geq 0\right\}\right]_{L_{l o c, w}^{2}\left([0, \infty) ; V_{\text {div }}^{\prime}\right)},
$$

where $[\cdot]_{X}$ denotes the closure in the space $X$, is compact in $L_{l o c, w}^{2}\left([0, \infty) ; V_{d i v}^{\prime}\right)$ (see, e.g., [12, Section 6] and [11, Proposition 6.8]).

As symbol space $\Sigma$ we take the compact metric space given by $\Sigma=\mathcal{H}_{+}\left(h_{0}\right)$. Recall that every $h \in \mathcal{H}_{+}\left(h_{0}\right)$ is translation compact in $L_{l o c, w}^{2}\left([0, \infty) ; V_{d i v}^{\prime}\right)$ as well (see [11, Proposition 6.9]) and

$$
\|h\|_{L_{t b}^{2}\left(0, \infty ; V_{d i v}^{\prime}\right)} \leq\left\|h_{0}\right\|_{L_{t b}^{2}\left(0, \infty ; V_{d i v}^{\prime}\right)}, \quad \forall h \in \mathcal{H}_{+}\left(h_{0}\right) .
$$

Hence we can state the main result of this section.

THEOREM 4. Let (A1)-(A7) hold and assume $h_{0} \in L_{t b}^{2}\left(0, \infty ; V_{d i v}^{\prime}\right)$. Then, the translation semigroup $\{T(t)\}$ acting on $\mathcal{K}_{\mathcal{H}_{+}\left(h_{0}\right)}$ possesses the uniform (w.r.t. $\left.h \in \mathcal{K}_{\mathcal{H}_{+}\left(h_{0}\right)}^{+}\right)$trajectory attractor $\mathcal{A}_{\mathcal{H}_{+}\left(h_{0}\right)}$. This set is strictly invariant, bounded in $\mathcal{F}_{b}^{+}$and compact in $\Theta_{\text {loc }}^{+}$. In addition, if the potential $F$ is bounded on $(-1,1)$ and $h_{0} \in L_{t b}^{2}\left(0, \infty ; G_{d i v}\right)$ or $h_{0}$ is translation-compact in $L_{\text {loc }}^{2}\left([0, \infty) ; V_{d i v}^{\prime}\right)$,

then $\mathcal{K}_{\mathcal{H}_{+}\left(h_{0}\right)}^{+}$is closed in $\Theta_{\text {loc }}^{+}, \mathcal{A}_{\mathcal{H}_{+}\left(h_{0}\right)} \subset \mathcal{K}_{\mathcal{H}_{+}\left(h_{0}\right)}^{+}$and we have

$$
\mathcal{A}_{\mathcal{H}_{+}\left(h_{0}\right)}=\mathcal{A}_{\omega\left(\mathcal{H}_{+}\left(h_{0}\right)\right)} \text {. }
$$

The proof of Theorem 4 is based on two propositions. The first one establishes a dissipative estimate of the form (5.1) for weak solutions to (1.1)-(1.5).

Proposition 5. Let (A1)-(A7) hold and let $h_{0} \in L_{t b}^{2}\left(0, \infty ; V_{d i v}^{\prime}\right)$. Then, for all $h \in \mathcal{H}_{+}\left(h_{0}\right)$, we have $\mathcal{K}_{h}^{+} \subset \mathcal{F}_{b}^{+}$and the following dissipative estimate holds

$$
d_{\mathcal{F}_{b}^{+}}(T(t) z, 0) \leq \Lambda_{0}\left(d_{\mathcal{F}_{b}^{+}}(z, 0)\right) e^{-k t}+\Lambda_{1}, \quad \forall t \geq 1,
$$


for all $z \in \mathcal{K}_{h}^{+}$. Here $\Lambda_{0}:[0, \infty) \rightarrow[0, \infty)$ is a nonnegative monotone increasing continuous function, $k$ and $\Lambda_{1}$ are two positive constants with $k=\min \left(1 / 2, \lambda_{1} \nu_{1}\right)$, $\lambda_{1}$ being the first eigenvalue of the Stokes operator $S$. Moreover, $\Lambda_{0}, \Lambda_{1}$ depend on $\nu_{1}, \nu_{2}, \lambda_{1}, F, J,|\Omega|$, and $\Lambda_{1}$ also depends on $\left\|h_{0}\right\|_{L_{t b}^{2}\left(0, \infty ; V_{d i v}^{\prime}\right)}$ and on $m_{0}$.

Proof. The following estimate can be obtained by arguing as in the proof of $[\mathbf{1 4}$, Corollary 2] (see also the proof of $[\mathbf{2 1}$, Theorem 5]). There exist two positive constants $k_{1}, k_{2}$ such that

$$
\mathcal{E}(z) \leq k_{1}\left(\frac{\nu_{1}}{2}\|\nabla v\|^{2}+\|\nabla \mu\|^{2}\right)+k_{2},
$$

for every weak solution $z=[v, \psi]$ to (1.1)-(1.5) satisfying $\bar{\psi}=0$. Furthermore, it can be shown that $k_{1}=\max \left(2,1 / \lambda_{1} \nu_{1}\right)$.

Take now $z=[v, \psi] \in \mathcal{K}_{h}^{+}$with $h \in \mathcal{H}_{+}\left(h_{0}\right)$ and set $\widetilde{z}=[v, \widetilde{\psi}]$, where $\widetilde{\psi}:=\psi-\bar{\psi}$. Recall that $\bar{\psi}=\overline{\psi_{0}}$. It is easily seen that $\widetilde{z}$ is a weak solution to the same system where the potential $F$ and the viscosity $\nu$ are replaced by, respectively,

$$
\widetilde{F}(s):=F\left(s+\bar{\psi}_{0}\right)-F\left(\overline{\psi_{0}}\right), \quad \widetilde{\nu}(s):=\nu\left(s+\overline{\psi_{0}}\right) .
$$

Since $z$ satisfies (2.13) for all $t \geq s$ and for a.a. $s \in(0, \infty)$, then an energy inequality of the same form as (2.13) also holds for $\widetilde{z}$, namely,

$$
\widetilde{\mathcal{E}}(\widetilde{z}(t))+\int_{s}^{t}\left(2\|\sqrt{\widetilde{\nu}(\widetilde{\psi})} D v\|^{2}+\|\nabla \widetilde{\mu}\|^{2}\right) d \tau \leq \widetilde{\mathcal{E}}(\widetilde{z}(s))+\int_{s}^{t}\langle h(\tau), v(\tau)\rangle d \tau,
$$

for all $t \geq s$ and for a.a. $s \in(0, \infty)$, where we have set

$$
\widetilde{\mathcal{E}}(\widetilde{z}(t)):=\frac{1}{2}\|v(t)\|^{2}+\frac{1}{4} \int_{\Omega} \int_{\Omega} J(x-y)(\widetilde{\psi}(x, t)-\widetilde{\psi}(y, t))^{2} d x d y+\int_{\Omega} \widetilde{F}(\widetilde{\psi}(t))
$$

and $\widetilde{\mu}:=a \widetilde{\psi}-J * \widetilde{\psi}+\widetilde{F}^{\prime}(\widetilde{\psi})=a \psi-J * \psi+F^{\prime}(\psi)=\mu$. The weak solution $\widetilde{z}$ fulfills $(\widetilde{\psi}, 1)=0$ and therefore (5.6) can be applied to $\widetilde{z}$. Such estimate and (5.7) entail the inequality

$$
\begin{aligned}
& \widetilde{\mathcal{E}}(\widetilde{z}(t))+\frac{1}{k_{1}} \int_{0}^{t} \widetilde{\mathcal{E}}(\widetilde{z}(\tau)) d \tau \leq \frac{k_{2}}{k_{1}}(t-s)+\frac{1}{2 \nu_{1}} \int_{s}^{t}\|h(\tau)\|_{V_{d i v}^{\prime}}^{2} d \tau \\
& +\widetilde{\mathcal{E}}(\widetilde{z}(s))+\frac{1}{k_{1}} \int_{0}^{s} \widetilde{\mathcal{E}}(\widetilde{z}(\tau)) d \tau, \quad \forall t \geq s, \quad \text { a.a. } s \in(0, \infty) .
\end{aligned}
$$

By means of the identity

$$
\widetilde{\mathcal{E}}(\widetilde{z}(t))=\mathcal{E}(z(t))-F\left(\overline{\psi_{0}}\right)|\Omega|,
$$

from the previous inequality we get

$$
\begin{aligned}
& \mathcal{E}(z(t))+k \int_{0}^{t} \mathcal{E}(z(\tau)) d \tau \leq l(t-s) \\
& +\frac{1}{2 \nu_{1}} \int_{s}^{t}\|h(\tau)\|_{V_{d i v}^{\prime}}^{2} d \tau+\mathcal{E}(z(s))+k \int_{0}^{s} \mathcal{E}(z(\tau)) d \tau
\end{aligned}
$$


for all $t \geq s$ and for a.a. $s \in(0, \infty)$, where $k=1 / k_{1}$ and $l=k_{2} / k_{1}+F\left(\overline{\psi_{0}}\right)|\Omega| / k_{1}$. By applying [21, Lemma 1] from (5.8) we deduce that

$$
\begin{aligned}
& \mathcal{E}(z(t)) \leq \mathcal{E}(z(s)) e^{-k(t-s)}+\frac{1}{2 \nu_{1}} \int_{s}^{t} e^{-k(t-\tau)}\left(\|h(\tau)\|_{V_{d i v}^{\prime}}^{2}+2 \nu_{1} l\right) d \tau \\
& \leq e^{k} \sup _{s \in(0,1)} \mathcal{E}(z(s)) e^{-k t}+K^{2},
\end{aligned}
$$

for all $t \geq 1$, where

$$
K^{2}=\frac{l}{k}+\frac{l}{2 \nu_{1}\left(1-e^{-k}\right)}\left\|h_{0}\right\|_{L_{t b}^{2}\left(0, \infty ; V_{d i v}^{\prime}\right)}^{2} .
$$

Here we have used (5.4). Note that $\left|\overline{\psi_{0}}\right| \leq m_{0}$ and therefore $K$ can be estimated by a constant depending on $\nu_{1}, \lambda_{1}, F, J,|\Omega|$ and on $h_{0}, m_{0}$. Observe now that we have

$$
\begin{aligned}
& C_{1}\left(\|v(s)\|^{2}+\|\psi(s)\|_{L^{2+2 q}(\Omega)}^{2+2 q}+\int_{\Omega} F(\psi(s))-1\right) \\
& \leq \mathcal{E}(z(s)) \leq C_{2}\left(\|v(s)\|^{2}+\|\psi(s)\|_{L^{2+2 q}(\Omega)}^{2+2 q}+\int_{\Omega} F(\psi(s))+1\right),
\end{aligned}
$$

and therefore

$$
\begin{aligned}
& \sup _{s \in(0,1)} \mathcal{E}(z(s)) \leq C_{2}\left(\|v\|_{L^{\infty}\left(0,1 ; G_{d i v}\right)}^{2}+\|\psi\|_{L^{\infty}\left(0,1 ; L^{2+2 q}(\Omega)\right)}^{2+2 q}+\sup _{s \in(0,1)} \int_{\Omega} F(\psi(s))+1\right) \\
& (5.11) \quad \leq C_{3} d_{\mathcal{F}_{b}^{+}}^{2+2 q}(z, 0) .
\end{aligned}
$$

By combining (5.9) with (5.10) and (5.11) we get

$$
\|v(t)\|^{2}+\|\psi(t)\|_{L^{2+2 q}(\Omega)}^{2+2 q}+\int_{\Omega} F(\psi(t)) \leq c d_{\mathcal{F}_{b}^{+}}^{2+2 q}(z, 0) e^{-k t}+K^{2}+c, \quad \forall t \geq 1,
$$

which yields

$$
\begin{aligned}
& \|T(t) v\|_{L^{\infty}\left(0, \infty ; G_{d i v}\right)}^{2}+\|T(t) \psi\|_{L^{\infty}\left(0, \infty ; L^{2+2 q}(\Omega)\right)}^{2+2 q}+\left\|\int_{\Omega} F(T(t) \psi)\right\|_{L^{\infty}(0, \infty)} \\
& \leq c d_{\mathcal{F}_{b}^{+}}^{2+2 q}(z, 0) e^{-k t}+K^{2}+c, \quad \forall t \geq 1 .
\end{aligned}
$$

On account of the definition of the metric $d_{\mathcal{F}_{b}^{+}},(5.13)$ allows to estimate three terms on the left hand side of (5.5). The remaining four terms on the left hand side of (5.5) can be handled by performing the same kind of calculations done in the proof of [21, Proposition 7]. In particular, the two terms in the $L_{t b}^{2}\left(0, \infty ; V_{d i v}\right)$-norm of $T(t) v$ and in the $L_{t b}^{2}(0, \infty ; V)$-norm of $T(t) \psi$ can be estimated by writing the energy inequality between $t$ and $t+1$ and by using the estimate

$$
\|\nabla \mu\|^{2} \geq k_{3}\|\nabla \psi\|^{2}-k_{4}\|\psi\|^{2},
$$

where $k_{3}=c_{0}^{4} / 2$ and $k_{4}=2\|\nabla J\|_{L^{1}}^{2}$, with $c_{0}=\alpha+\beta+\min _{[-1,1]} F_{2}^{\prime \prime}>0$. This last estimate has been obtained in [14] for the case of regular potentials, but it still holds for singular potentials satisfying assumption (A6). Finally, the two terms in the $L_{t b}^{4 / 3}\left(0, \infty ; V_{d i v}^{\prime}\right)$-norm of $T(t) v_{t}$ and in the $L_{t b}^{2}\left(0, \infty ; V^{\prime}\right)$-norm of $T(t) \psi_{t}$ can 
be estimated by comparison on account of (5.12), using also the estimates for the $L_{t b}^{2}\left(0, \infty ; V_{d i v}\right)$-norm of $T(t) v$ and the $L_{t b}^{2}(0, \infty ; V)$-norm of $T(t) \psi$. We refer to $[\mathbf{2 1}$, Proposition 7] for the details.

The next proposition, which concerns with the $\left(\Theta_{M}, L^{2}\left(0, M ; V_{d i v}^{\prime}\right)\right)$-closedness property of the family $\left\{\mathcal{K}_{h}^{M}\right\}_{h \in L^{2}\left(0, M ; V_{d i v}^{\prime}\right)}$ of trajectory spaces on $[0, M]$, requires a boundedness assumption on the potential $F$.

Proposition 6. Let (A1)-(A7) hold and assume that the potential $F$ is bounded on $(-1,1)$. Let $h_{m} \in L^{2}\left(0, M ; V_{d i v}^{\prime}\right)$ and consider $\left[v_{m}, \psi_{m}\right] \in \mathcal{K}_{h_{m}}^{M}$ such that $\left\{\left[v_{m}, \psi_{m}\right]\right\}$ converges to $[v, \psi]$ in $\Theta_{M}$ and $\left\{h_{m}\right\}$ converges to $h$ strongly in

$$
L^{2}\left(0, M ; V_{d i v}^{\prime}\right) \text {. }
$$

Then $[v, \psi] \in \mathcal{K}_{h}^{M}$.

Proof. Observe that $\left[v_{m}, \psi_{m}\right] \in \mathcal{K}_{h_{m}}^{+}$

(i) belongs to $\mathcal{F}_{M}$ with $\mu_{m}$ satisfying (2.8);

(ii) fulfills (2.10)-(2.11) together with $\mu_{m}=a \psi_{m}-J * \psi_{m}+F^{\prime}\left(\psi_{m}\right)$ and $h=h_{m}$;

(iii) satisfies the energy inequality

$$
\begin{aligned}
& \mathcal{E}\left(z_{m}(t)\right)+\int_{s}^{t}\left(2\left\|\sqrt{\nu\left(\psi_{m}\right)} D v_{m}\right\|^{2}+\left\|\nabla \mu_{m}\right\|^{2}\right) d \tau \\
& \leq \mathcal{E}\left(z_{m}(s)\right)+\int_{s}^{t}\left\langle h_{m}(\tau), v_{m}(\tau)\right\rangle d \tau,
\end{aligned}
$$

for each $m \in \mathbb{N}$, for a.a. $s \in[0, M]$ and for all $t \in[0, M]$ with $t \geq s$. Thus, due to the convergence assumption on the sequence $\left\{\left[v_{m}, \psi_{m}\right]\right\}$ and to the boundedness of $F$, it is immediate to see that there exists a constant $c>0$ such that

$$
\left|\mathcal{E}\left(z_{m}(s)\right)\right| \leq c, \quad \forall m \text {, a.a. } s \in[0, M] .
$$

Therefore, (5.14) and the convergence assumption on the sequence $\left\{h_{m}\right\}$ imply the control $\left\|\nabla \mu_{m}\right\|_{L^{2}(0, M ; H)} \leq c$. On the other hand, by exploiting the argument used in the proof of Theorem 1 it is easy to find the bound

$$
\left\|F^{\prime}\left(\psi_{m}\right)\right\|_{L^{2}\left(0, T ; L^{1}(\Omega)\right)} \leq L\left(\overline{\psi_{m}}\right),
$$

with some constant $L\left(\overline{\psi_{m}}\right)$ depending on $\overline{\psi_{m}}$, and furthermore we also have $\left|\overline{\psi_{m}}\right| \leq$ $m_{0}$, with $m_{0} \in(0,1)$. Therefore, noting that $\int_{\Omega} \mu_{m}=\int_{\Omega} F^{\prime}\left(\psi_{m}\right)$, we deduce that $\left\|\overline{\mu_{m}}\right\|_{L^{2}(0, M)} \leq c$, with the constant $c$ depending on the fixed parameter $m_{0}$. The Poincaré-Wirtinger inequality then implies

$$
\left\|\mu_{m}\right\|_{L^{2}(0, M ; V)} \leq c .
$$

As a consequence, there exists $\mu \in L^{2}(0, M ; V)$ such that up to a subsequence we have

$$
\mu_{m} \rightarrow \mu, \quad \text { weakly in } L^{2}(0, M ; V) .
$$


Since, as a consequence of the convergence assumption on $\left\{\left[v_{m}, \psi_{m}\right]\right\}$, for a subsequence we have $\left[v_{m}, \psi_{m}\right] \rightarrow[v, \psi]$ strongly in $L^{2}\left(0, M ; G_{d i v} \times H\right)$ and hence $\psi_{m} \rightarrow \psi$ also almost everywhere in $\Omega \times(0, M)$, then we get $\mu=a \psi-J * \psi+F^{\prime}(\psi)$. Using now the convergence assumptions on $\left\{\left[v_{m}, \psi_{m}\right]\right\}$ and on $\left\{h_{m}\right\}$, the above mentioned strong convergence and (5.17), we can pass to the limit in the variational formulation for the weak solution $\left[v_{m}, \psi_{m}\right]$ with external force $h_{m}$ and deduce that $[v, \psi]$ is a weak solution with external force $h$.

Finally, in order to prove that the weak solution $[v, \psi]$ satisfies the energy inequality on $[0, M]$ with external force $h$ we let $m \rightarrow \infty$ in (5.14). In particular, we rely on the convergence $\sqrt{\nu\left(\psi_{m}\right)} D v_{m} \rightarrow \sqrt{\nu(\psi)} D v$ weakly in $L^{2}(0, M ; H)$ (cf. (3.53)) and on Lebesgue's theorem to pass to the limit in the nonlinear term $\int_{\Omega} F\left(\psi_{m}(s)\right)$. Hence we conclude that $[v, \psi] \in \mathcal{K}_{h}^{M}$.

Remark 9. It is not difficult to see, by arguing as in [12, Chap. XV, Prop. 1.1], that the same conclusion of Proposition 6 holds if the convergence assumption on $\left\{h_{m}\right\}$ is replaced with the weak convergence $h_{m} \rightarrow h$ in $L^{2}\left(0, M ; G_{d i v}\right)$.

Proof of Theorem 4. In virtue of Proposition 5 the ball $B_{\mathcal{F}_{b}^{+}}\left(0,2 \Lambda_{0}\right):=$ $\left\{z \in \mathcal{F}_{b}^{+}: d_{\mathcal{F}_{b}^{+}}(z, 0) \leq 2 \Lambda_{0}\right\}$ is a uniformly (w.r.t. $h \in \mathcal{H}_{+}\left(h_{0}\right)$ ) absorbing set for the family $\left\{\mathcal{K}_{h}^{+}\right\}_{h \in \mathcal{H}_{+}\left(h_{0}\right)}$. Such a ball is also precompact in $\Theta_{\text {loc }}^{+}$. By applying the first part of Theorem 3 we deduce the existence of the uniform (w.r.t. $h \in \mathcal{H}_{+}\left(h_{0}\right)$ ) trajectory attractor $\mathcal{A}_{\mathcal{H}_{+}\left(h_{0}\right)} \subset B_{\mathcal{F}_{b}^{+}}\left(0,2 \Lambda_{0}\right)$, which is compact in $\Theta_{l o c}^{+}$and, since $T(t)$ is continuous in $\Theta_{l o c}^{+}$, strictly invariant. Proposition 6 and the fact that $\mathcal{H}_{+}\left(h_{0}\right)$ is a compact metric space imply that the united trajectory space $\mathcal{K}_{\mathcal{H}_{+}\left(h_{0}\right)}^{+}$is closed in $\Theta_{l o c}^{+}$. The second part of Theorem 3 allows us to conclude the proof.

\section{Further properties of the trajectory attractor}

Let us discuss first some structural properties of the trajectory attractor.

Denote by $Z\left(h_{0}\right):=Z\left(\mathcal{H}_{+}\left(h_{0}\right)\right)$ the set of all complete symbols in $\omega\left(\mathcal{H}_{+}\left(h_{0}\right)\right)$. Recall that a function $\zeta: \mathbb{R} \rightarrow V_{\text {div }}^{\prime}$ with $\zeta \in L_{\text {loc }}^{2}\left(\mathbb{R} ; V_{\text {div }}^{\prime}\right)$ is a complete symbol in $\omega\left(\mathcal{H}_{+}\left(h_{0}\right)\right)$ if $\Pi_{+} T(t) \zeta \in \omega\left(\mathcal{H}_{+}\left(h_{0}\right)\right)$ for all $t \in \mathbb{R}$, where $\Pi_{+}$is the restriction operator on the semiaxis $[0, \infty)$. It can be proved (see $[\mathbf{1 1}$, Section 4$]$ or $[\mathbf{1 2}$, Chap. XIV, Section 2]) that, due to the strict invariance of $\omega\left(\mathcal{H}_{+}\left(h_{0}\right)\right)$, given a symbol $h \in \omega\left(\mathcal{H}_{+}\left(h_{0}\right)\right)$ there exists at least one complete symbol $\widehat{h}$ (not necessarily unique) which is an extension of $h$ on $(-\infty, 0]$ and such that $\Pi_{+} T(t) \widehat{h} \in \omega\left(\mathcal{H}_{+}\left(h_{0}\right)\right)$ for all $t \in \mathbb{R}$. Note that we have $\Pi_{+} Z\left(h_{0}\right)=\omega\left(\mathcal{H}_{+}\left(h_{0}\right)\right)$.

To every complete symbol $\zeta \in Z\left(h_{0}\right)$ there corresponds by [12, Chap. XIV, Definition 2.5] (see also [11, Definition 4.4]) the kernel $\mathcal{K}_{\zeta}$ in $\mathcal{F}_{b}$ which consists of the union of all complete trajectories which belong to $\mathcal{F}_{b}$, i.e., all weak solutions $z=[v, \psi]: \mathbb{R} \rightarrow G_{d i v} \times H$ with external force $\zeta \in Z\left(h_{0}\right)$ (in the sense of Definition 1 with $T \in \mathbb{R}$ ) satisfying (2.13) on $\mathbb{R}$ (i.e., for all $t \geq s$ and for a.a. $s \in \mathbb{R}$ ) that 
belong to $\mathcal{F}_{b}$. We recall that the space $\left(\mathcal{F}_{b}, d_{\mathcal{F}_{b}}\right)$ is defined as the space $\left(\mathcal{F}_{b}^{+}, d_{\mathcal{F}_{b}}^{+}\right)$ with the time interval $(0, \infty)$ replaced by $\mathbb{R}$ in the definitions of $\mathcal{F}_{b}^{+}$and $d_{\mathcal{F}_{b}^{+}}$. The space $\left(\mathcal{F}_{l o c}, \Theta_{l o c}\right)$ can be defined in the same way.

Set

$$
\mathcal{K}_{Z\left(h_{0}\right)}:=\bigcup_{\zeta \in Z\left(h_{0}\right)} \mathcal{K}_{\zeta} .
$$

Then, if the assumptions of Theorem 4 hold with $F$ bounded in $(-1,1)$ and $h_{0} \in$ $L_{t b}^{2}\left(0, \infty ; G_{d i v}\right)$ or $h_{0}$ is translation-compact in $L_{l o c}^{2}\left([0, \infty) ; V_{d i v}^{\prime}\right)$, we also have (see, e.g., [11, Theorem 4.1])

$$
\mathcal{A}_{\mathcal{H}_{+}\left(h_{0}\right)}=\mathcal{A}_{\omega\left(\mathcal{H}_{+}\left(h_{0}\right)\right)}=\Pi_{+} \mathcal{K}_{Z\left(h_{0}\right)},
$$

and the set $\mathcal{K}_{Z\left(h_{0}\right)}$ is compact in $\Theta_{\text {loc }}$ and bounded in $\mathcal{F}_{b}$.

On the other hand, it is not difficult to see that, under the assumptions of Theorem $4, \mathcal{K}_{\zeta} \neq \emptyset$ for all $\zeta \in Z\left(h_{0}\right)$. Indeed, by virtue of [11, Theorem 4.1] (see also [12, Chap. XIV, Theorem 2.1]), this is a consequence of the fact that the family $\left\{\mathcal{K}_{h}^{+}\right\}_{h \in \mathcal{H}_{+}\left(h_{0}\right)}$ of trajectory spaces satisfies the following condition: there exists $R>0$ such that $B_{\mathcal{F}_{b}^{+}}(0, R) \cap \mathcal{K}_{h}^{+} \neq \emptyset$ for all $h \in \mathcal{H}_{+}\left(h_{0}\right)$. In order to check this condition fix an initial datum $z_{0}^{*}=\left[v_{0}^{*}, \psi_{0}^{*}\right]$, with $v_{0}^{*}, \psi_{0}^{*}$ taken as in Theorem 1. We know that for every $h \in \mathcal{H}_{+}\left(h_{0}\right)$ there exists a trajectory $z_{h}^{*} \in \mathcal{K}_{h}^{+}$such that $z_{h}^{*}(0)=z_{0}^{*}$ and such that the energy inequality (2.13) holds for all $t \geq s$ and for a.a. $s \in(0, \infty)$, including $s=0$. Arguing as in Proposition 5 (cf. (5.9) written for $s=0$ and all $t \geq 0)$ we get an estimate of the form $d_{\mathcal{F}_{b}^{+}}\left(z_{h}^{*}, 0\right) \leq \Lambda\left(z_{0}^{*}, h_{0}\right)$ (see also (5.4)), where the positive constant $\Lambda$ depends on $\mathcal{E}\left(z_{0}^{*}\right)$ and on the norm $\left\|h_{0}\right\|_{L_{t b}^{2}\left(0, \infty ; V_{d i v}^{\prime}\right)}$. The above condition is thus fulfilled by choosing $R=\Lambda\left(z_{0}^{*}, h_{0}\right)$.

As far as the attraction properties are concerned, we observe that, due to compactness results, the trajectory attractor attracts the subsets of the family $\mathcal{B}_{\mathcal{H}_{+}\left(h_{0}\right)}^{+}$in some strong topologies. Indeed, setting

$$
\mathbb{X}_{\delta_{1}, \delta_{2}}:=H^{\delta_{1}}(\Omega)^{d} \times H^{\delta_{2}}(\Omega), \quad \mathbb{Y}_{\delta_{1}, \delta_{2}}:=H^{-\delta_{1}}(\Omega)^{d} \times\left(H^{\delta_{2}}(\Omega)\right)^{\prime},
$$

where $0 \leq \delta_{1}, \delta_{2}<1$ and using the compact embeddings

$$
\begin{aligned}
& L^{2}\left(0, M ; V_{d i v} \times V\right) \cap W^{1,4 / 3}\left(0, M ; V_{d i v}^{\prime} \times V^{\prime}\right) \hookrightarrow \hookrightarrow L^{2}\left(0, M ; \mathbb{X}_{\delta_{1}, \delta_{2}}\right), \\
& L^{\infty}\left(0, M ; G_{d i v} \times H\right) \cap W^{1,4 / 3}\left(0, M ; V_{d i v}^{\prime} \times V^{\prime}\right) \hookrightarrow \hookrightarrow C\left([0, M] ; \mathbb{Y}_{\delta_{1}, \delta_{2}}\right),
\end{aligned}
$$

then Theorem 4 implies the following (see [12, Chap. XIV, Theorem 2.2])

Corollary 1. Let (A1)-(A7) hold and assume $h_{0} \in L_{t b}^{2}\left(0, \infty ; V_{d i v}^{\prime}\right)$. Then,

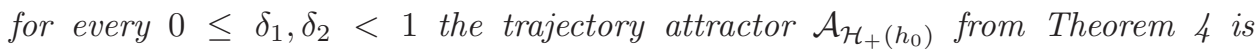
compact in $L_{\text {loc }}^{2}\left([0, \infty) ; \mathbb{X}_{\delta_{1}, \delta_{2}}\right) \cap C\left([0, \infty) ; \mathbb{Y}_{\delta_{1}, \delta_{2}}\right)$, bounded in $\left.L_{t b}^{2}(0, \infty) ; \mathbb{X}_{\delta_{1}, \delta_{2}}\right) \cap$ $C_{b}\left([0, \infty) ; \mathbb{Y}_{\delta_{1}, \delta_{2}}\right)$, and for every $B \in \mathcal{B}_{\mathcal{H}_{+}\left(h_{0}\right)}^{+}$and every $M>0$ we have

$$
\operatorname{dist}_{L^{2}\left(0, M ; \mathbb{X}_{\delta_{1}, \delta_{2}}\right)}\left(\Pi_{[0, M]} T(t) B, \Pi_{[0, M]} \mathcal{A}_{\mathcal{H}_{+}\left(h_{0}\right)}\right) \rightarrow 0,
$$




$$
\operatorname{dist}_{C\left([0, M] ; \mathbb{Y}_{\delta_{1}, \delta_{2}}\right)}\left(\Pi_{[0, M]} T(t) B, \Pi_{[0, M]} \mathcal{A}_{\mathcal{H}_{+}\left(h_{0}\right)}\right) \rightarrow 0,
$$

as $t \rightarrow+\infty$, where dist $_{X}(A, B)$ denotes the Hausdorff semidistance in the Banach space $X$ between $A, B \subset X$, and $\Pi_{[0, M]}$ is the restriction operator to the interval $[0, M]$.

Let us now define, for every $B \subset \mathcal{K}_{\mathcal{H}_{+}\left(h_{0}\right)}^{+}$, the sections

$$
B(t):=\{[v(t), \psi(t)]:[v, \psi] \in B\} \subset \mathbb{Y}_{\delta_{1}, \delta_{2}}, \quad t \geq 0 .
$$

Similarly we set

$$
\begin{aligned}
& \mathcal{A}_{\mathcal{H}_{+}\left(h_{0}\right)}(t):=\left\{[v(t), \psi(t)]:[v, \psi] \in \mathcal{A}_{\left.\mathcal{H}_{+}\left(h_{0}\right)\right\} \subset \mathbb{Y}_{\delta_{1}, \delta_{2}}, \quad t \geq 0}\right. \\
& \mathcal{K}_{Z\left(h_{0}\right)}(t):=\left\{[v(t), \psi(t)]:[v, \psi] \in \mathcal{K}_{Z\left(h_{0}\right)}\right\} \subset \mathbb{Y}_{\delta_{1}, \delta_{2}}, \quad t \in \mathbb{R} .
\end{aligned}
$$

Then, as a further consequence of Theorem 4 we have (see [12, Chap. XIV, Definition 2.6, Corollary 2.2]) the following

Corollary 2. Let (A1)-(A7) hold and assume $h_{0} \in L_{t b}^{2}\left(0, \infty ; G_{\text {div }}\right)$ or $h_{0}$ translation-compact in $L_{l o c}^{2}\left([0, \infty) ; V_{d i v}^{\prime}\right)$. Then the bounded subset

$$
\mathcal{A}_{g l}:=\mathcal{A}_{\mathcal{H}_{+}\left(h_{0}\right)}(0)=\mathcal{K}_{Z\left(h_{0}\right)}(0)
$$

is the uniform (w.r.t. $\left.h \in \mathcal{H}_{+}\left(h_{0}\right)\right)$ global attractor in $\mathbb{Y}_{\delta_{1}, \delta_{2}}, 0 \leq \delta_{1}, \delta_{2}<1$, of system (1.1)-(1.5), namely (i) $\mathcal{A}_{g l}$ is compact in $\mathbb{Y}_{\delta_{1}, \delta_{2}}$, (ii) $\mathcal{A}_{\text {gl }}$ satisfies the attracting property

$$
\operatorname{dist}_{\mathbb{Y}_{\delta_{1}, \delta_{2}}}\left(B(t), \mathcal{A}_{g l}\right) \rightarrow 0, \quad t \rightarrow+\infty
$$

for every $B \in \mathcal{B}_{\mathcal{H}_{+}\left(h_{0}\right)}$, and (iii) $\mathcal{A}_{g l}$ is the minimal set satisfying (i) and (ii).

REMARK 10. In the 2D case the energy identity might be exploited to show the convergence to the trajectory attractor in the strong topology of the original phase space. This was done in [13] for a reaction-diffusion system without uniqueness.

Acknowledgments. The authors thank the reviewers for their careful reading as well as for their pertinent remarks and suggestions. This work was partially supported by the Italian MIUR-PRIN Research Project 2008 "Transizioni di fase, isteresi e scale multiple". The first author was also supported by the FTP7-IDEASERC-StG Grant $\sharp 200497$ (BioSMA) and the FP7-IDEAS-ERC-StG Grant \#256872 (EntroPhase). 


\section{References}

[1] H. Abels, On a diffusive interface model for two-phase flows of viscous, incompressible fluids with matched densities, Arch. Ration. Mech. Anal. 194 (2009), 463-506.

[2] H. Abels, Longtime behavior of solutions of a Navier-Stokes/Cahn-Hilliard system, Proceedings of the Conference "Nonlocal and Abstract Parabolic Equations and their Applications", Bedlewo, Banach Center Publ. 86 (2009), 9-19.

[3] H. Abels, M. Röger, Existence of weak solutions for a non-classical sharp interface model for a two-phase flow of viscous, incompressible fluids, Ann. Inst. H. Poincaré Anal. Non Linéaire 26 (2009), 2403-2424.

[4] H. Abels, M. Wilke, Convergence to equilibrium for the Cahn-Hilliard equation with a logarithmic free energy, Nonlinear Anal. 67 (2007), 3176-3193.

[5] J.M. Ball, Continuity properties and global attractors of generalized semiflows and the Navier-Stokes equation, J. Nonlinear Sci. 7 (1997), 475-502 (Erratum, J. Nonlinear Sci. 8 (1998), 233).

[6] V. Barbu, Nonlinear Semigroups and Differential Equations in Banach Spaces, Noordhoff, Leyden, 1976.

[7] P.W. Bates, J. Han, The Dirichlet boundary problem for a nonlocal Cahn-Hilliard equation, J. Math. Anal. Appl. 311 (2005), 289-312.

[8] P.W. Bates, J. Han, The Neumann boundary problem for a nonlocal Cahn-Hilliard equation, J. Differential Equations 212 (2005), 235-277.

[9] F. Boyer, Mathematical study of multi-phase flow under shear through order parameter formulation, Asymptot. Anal. 20 (1999), 175-212.

[10] J.W. Cahn, J.E. Hilliard, Free energy of a nonuniform system. I. Interfacial free energy, J. Chem. Phys. 28 (1958), 258-267.

[11] V.V. Chepyzhov, M.I. Vishik, Evolution equations and their trajectory attractors, J. Math. Pures Appl. 76 (1997), 913-964.

[12] V.V. Chepyzhov, M.I. Vishik, Attractors for Equations of Mathematical Physics, Amer. Math. Soc. Colloq. Publ., vol. 49, American Mathematical Society, Providence, RI, 2002.

[13] V.V. Chepyzhov, M.I. Vishik, S.V. Zelik, Strong trajectory attractor for a dissipative reactiondiffusion system, Doklady Mathematics 82 (2010) 869-873.

[14] P. Colli, S. Frigeri, M. Grasselli, Global existence of weak solutions to a nonlocal CahnHilliard-Navier-Stokes system, J. Math. Anal. Appl. 386 (2012), 428-444.

[15] P. Colli, G. Gilardi, M. Grasselli, G. Schimperna, The conserved phase-field system with memory, Advances Math. Sci. Appl. 11 (2001), 265-291.

[16] P. Colli, P. Krejčí, E. Rocca, J. Sprekels, Nonlinear evolution inclusions arising from phase change models, Czechoslovak Math. J. 57 (2007), 1067-1098.

[17] A. Debussche, L. Dettori, On the Cahn-Hilliard equation with a logarithmic free energy, Nonlinear Anal. 24 (1995), 1491-1514.

[18] C.M. Elliott, H. Garcke, On the Cahn-Hilliard equation with degenerate mobility, SIAM J. Math. Anal. 27 (1996), 404-423.

[19] C.M. Elliott, S. Luckhaus, A generalised diffusion equation for phase separation of a multicomponent mixture with interfacial free energy, IMA Preprint Series, 887, 1991.

[20] C. Foias, R. Temam, The connection between the Navier-Stokes equations, dynamical systems, and turbulence theory, Proc. Directions in Partial Differential Equations, Madison, WI, 1985, Publ. Math. Res. Center Univ. Wisconsin 54, Academic Press, Boston, MA, 1987, 55-73.

[21] S. Frigeri, M. Grasselli, Global and trajectories attractors for a nonlocal Cahn-HilliardNavier-Stokes system, J. Dynam. Differential Equations, DOI 10.1007/s10884-012-9272-3. 
[22] C.G. Gal, M. Grasselli, Asymptotic behavior of a Cahn-Hilliard-Navier-Stokes system in 2D, Ann. Inst. H. Poincaré Anal. Non Linéaire 27 (2010), 401-436.

[23] C.G. Gal, M. Grasselli, Trajectory attractors for binary fluid mixtures in 3D, Chinese Ann. Math. Ser. B 31 (2010), 655-678.

[24] G. Giacomin, J.L. Lebowitz, Phase segregation dynamics in particle systems with long range interactions. I. Macroscopic limits, J. Statist. Phys. 87 (1997), 37-61.

[25] G. Giacomin, J.L. Lebowitz, Phase segregation dynamics in particle systems with long range interactions. II. Phase motion, SIAM J. Appl. Math. 58 (1998), 1707-1729.

[26] M.E. Gurtin, D. Polignone, J. Viñals, Two-phase binary fluids and immiscible fluids described by an order parameter, Math. Models Meth. Appl. Sci. 6 (1996), 8-15.

[27] B. Haspot, Existence of global weak solution for compressible fluid models with a capillary tensor for discontinuous interfaces, Differential Integral Equations 23 (2010), 899-934.

[28] P.C. Hohenberg, B.I. Halperin, Theory of dynamical critical phenomena, Rev. Mod. Phys. 49 (1977), 435-479.

[29] M. Heida, J. Málek, K.R. Rajagopal, On the development and generalizations of CahnHilliard equations within a thermodynamic framework, Z. Angew. Math. Phys. 63 (2012), 145-169.

[30] D. Jasnow, J. Viñals, Coarse-grained description of thermo-capillary flow, Phys. Fluids 8 (1996), 660-669.

[31] N. Kenmochi, M. Niezgódka, I. Pawlow, Subdifferential operator approach to the CahnHilliard equation with constraint, J. Differential Equations 117 (1995), 320-356.

[32] A. Morro, Phase-field models of Cahn-Hilliard Fluids and extra fluxes, Adv. Theor. Appl. Mech. 3 (2010), 409-424.

[33] C. Rohde, On local and non-local Navier-Stokes-Korteweg systems for liquid-vapour phase transitions, Z. Angew. Math. Mech. 85 (2005), 839-857.

[34] G.R. Sell, Global attractors for the three-dimensional Navier-Stokes equations, J. Dynam. Differential Equations 8 (1996), 1-33.

[35] V.N. Starovoitov, The dynamics of a two-component fluid in the presence of capillary forces, Math. Notes 62 (1997), 244-254.

[36] R. Temam, Navier-Stokes equations and nonlinear functional analysis, Second edition, CBMS-NSF Reg. Conf. Ser. Appl. Math. 66, SIAM, Philadelphia, PA, 1995.

[37] L. Zhao, H. Wu, H. Huang, Convergence to equilibrium for a phase-field model for the mixture of two viscous incompressible fluids, Commun. Math. Sci. 7 (2009), 939-962.

Dipartimento di Matematica F. Enriques, Università degli Studi di Milano, Milano I-20133, ITALY

E-mail address: sergio.frigeri@unimi.it

Dipartimento di Matematica F. Brioschi, Politecnico di Milano, Milano I-20133, ITALY

E-mail address: maurizio.grasselli@polimi.it 